\title{
Trajectory Design and Power Allocation for Drone-Assisted NR-V2X Network with Dynamic NOMA/OMA
}

\author{
Omid Abbasi, Student Member, IEEE, Halim Yanikomeroglu, Fellow, IEEE, Afshin Ebrahimi, Member, IEEE, and \\ Nader Mokari, Senior Member, IEEE
}

\begin{abstract}
In this paper, we find trajectory planning and power allocation for a vehicular network in which an unmanned-aerialvehicle (UAV) is considered as a relay to extend coverage for two disconnected far vehicles. We show that in a two-user network with an amplify-and-forward (AF) relay, non-orthogonalmultiple-access (NOMA) always has better or equal sum-rate in comparison to orthogonal-multiple-access (OMA) at high signalto-noise-ratio (SNR) regime. However, for the cases where i) base station (BS)-to-relay link is weak, or ii) two users have similar links, or iii) BS-to-relay link is similar to relay-to-weak user link, applying NOMA has negligible sum-rate gain. Hence, due to the complexity of successive-interference-cancellation (SIC) decoding in NOMA, we propose a dynamic NOMA/OMA scheme in which OMA mode is selected for transmission when applying NOMA has only negligible gain. Also, we show that OMA always has better min-rate than NOMA at high SNR regime. Further, we formulate two optimization problems which maximize the sum-rate and min-rate of the two vehicles. These problems are non-convex, and hence we propose an iterative algorithm based on alternating-optimization (AO) method which solves trajectory and power allocation sub-problems by successive-convexapproximation (SCA) and difference-of-convex (DC) methods, respectively. Finally, the above-mentioned performance is confirmed by simulations.
\end{abstract}

Index Terms-Non-orthogonal multiple access, aerial relaying, dynamic multiple access, trajectory design, power allocation, V2X.

\section{INTRODUCTION}

Unmanned aerial vehicle (UAV) communication has attracted substantial attention in the context of $5 \mathrm{G}$ and beyond$5 \mathrm{G}$ due to its many advantages such as high mobility, low cost, and on-demand deployment [1]. UAV-enabled communications have some advantages over terrestrial wireless communications. Due to the higher possibility of the existence of the lineof-sight (LOS) link between UAV and ground users [2], there is less path loss for these links. Also, multipath fading rarely

This work was supported in part by Huawei Canada Co., Ltd. (Corresponding author: Afshin Ebrahimi)

O. Abbasi and A. Ebrahimi are with the Department of Electrical Engineering, Sahand University of Technology, Tabriz, Iran. O. Abbasi is also with the Department of Systems and Computer Engineering, Carleton University, Ottawa, ON K1S5B6, Canada. (e-mail: om_abbasi@sut.ac.ir; omidabbasi@sce.carleton.ca; aebrahimi@sut.ac.ir)

H. Yanikomeroglu is with the Department of Systems and Computer Engineering, Carleton University, Ottawa, ON K1S5B6, Canada. (e-mail: halim@sce.carleton.ca)

N. Mokari is with the Department of Electrical and Computer Engineering, Tarbiat Modares University, Tehran, Iran. (e-mail: nader.mokari@modares.ac.ir) occurs in UAV-to-ground links. UAVs can be used as aerial base stations (BS) [3] and as relays [4] in practical scenarios that require on-demand deployment [5].

Vehicular communication has gained much attention in recent years due to its potential to enhance road safety and traffic efficiency [6]. Furthermore, providing communication for vehicles improves the quality of infotainment (information and entertainment) experience in vehicles [7]. This technology also facilitates emerging autonomous drivers. These applications require a considerable amount of data exchange, high capacity, and low latency. IEEE $802.11 \mathrm{p}$ is a standard which adds wireless access for vehicular environment (WAVE) [8] as dedicated-short-rang-communication (DSRC). However, IEEE $802.11 \mathrm{p}$ networks have coverage problem for vehicleto-infrastructure (V2I) connections [9]. Cellular-vehicle-toeverything (C-V2X) has advantages of high data rates and greater coverage than WAVE [10]. Recently, IEEE 802.11p and $\mathrm{C}-\mathrm{V} 2 \mathrm{X}$ technologies are updated to IEEE $802.11 \mathrm{bd}$ and new radio (NR)-V2X to satisfy high reliability and low latency requirements [11].

\section{A. Related Works}

Non-orthogonal multiple access (NOMA) [12] has attracted significant attention during 5G standardization [13]. Also, cooperative NOMA has been considered for terrestrial networks in recent years. In [14], near users acted as relay for far users. In [15], the authors proposed a new cooperative NOMA protocol in which near NOMA users act as energy harvesting relays to help far users. The authors in [16] studied the performance of a cooperative NOMA system with a dedicated full-duplex relay which worked in amplify-and-forward (AF) mode. The impact of relay selection on the performance of a cooperative NOMA system was studied in [17]. In [18], the authors exploited a decode-and-forward relay to send information to far users. The authors of [18] derived closedform expressions for outage probability and ergodic sum-rate of NOMA users. The authors in [19] proposed a cooperative relaying system based on NOMA which uses an AF relay.

In UAV-aided relay networks, UAVs are deployed to extend coverage and provide wireless connectivity for distant users without reliable direct communication links. In [20], an algorithm for energy efficiency maximization was proposed in which aerial relay moves in a circular trajectory. The authors in [4] considered a novel mobile relaying technique, where 
the relay nodes are mounted on UAVs. They studied the throughput maximization problem in mobile relaying systems by optimizing the source/relay transmit power along with the relay trajectory. In [5], the authors studied a network that consists of a BS and several aerial relays which serve several ground users. The deployment of UAVs for intercellular traffic offloading was studied in [21]. In [22], the authors considered a UAV relay network, where the UAV works as an AF relay. In [23], a UAV was employed as a relay to improve fairness and energy efficiency. In [24], the authors considered maximizing the throughput of a mobile relay system employing power allocation and UAV trajectory planning.

There are a few studies that exploit NOMA for a UAVmounted BS to serve terrestrial users [25]. In [26], the authors deployed a fixed-wing type UAV which moves in a circular trajectory around the centre of a macro-cell to provide coverage to the ground users with NOMA scheme. In [27], the authors proposed a power allocation scheme to maximize the sum-rate of the NOMA system by reducing energy expense for the UAV. Authors in [28], considered a multi-user system, in which a single-antenna UAV-BS serves a large number of ground users by employing NOMA. In [29], the placement and power allocation were jointly optimized to improve the performance of the NOMA-UAV network. In [30], a UAV and BS cooperate to serve ground users simultaneously. In this paper, the sum rate was maximized by jointly optimizing the UAV trajectory and the NOMA precoding.

Vehicular communication has attracted much attention in recent years. In [9], the authors investigated the spectrum sharing and power allocation design of device-to-device (D2D)enabled vehicular networks. In [10], two NOMA-based relayassisted broadcasting and multicasting schemes were proposed for $\mathrm{C}-\mathrm{V} 2 \mathrm{X}$ communications. In [31], the resource allocation problem for NOMA-enabled V2X communications was investigated. The authors in [32] introduced cache-aided NOMA as an enabling technology for vehicular networks.

\section{B. Motivations and Contributions}

To the best of our knowledge, there are no works in the related literature that have considered aerial communication for vehicular networks. In this paper, we consider a vehicular network in which a ground BS serves disconnected terrestrial vehicles with the aid of an aerial relay. Due to mitigating resource collision, NOMA reduces latency and therefore is a good choice for vehicular communication. Note that applying NOMA is straightforward for air-to-ground channels rather than for ground-to-ground ones. Indeed, multipath fading is not dominant in air-to-ground links and there is less randomness in these links. Hence, in order to apply NOMA scheme, we just need to sort the vehicles based on their distances.

In this paper, we show that in a two-user network with an AF relay, NOMA always has better or equal sum-rate performance in comparison to OMA at high SNR regime. However, for the cases where i) BS-to-relay link is weak, or ii) two users have similar links, or iii) BS-to-relay link is similar to relayto-weak user link, applying NOMA has negligible sum-rate gain. Hence, due to the complexity of successive-interferencecancellation (SIC) decoding in NOMA, we propose a dynamic
NOMA/OMA scheme in which OMA is selected for transmission when applying NOMA has negligible gain. In the proposed scheme, both vehicles can apply SIC based on the quality of the channel between these vehicles and the relay node. Also, we show that OMA always has better min-rate performance than NOMA at high SNR regime.

We consider two scenarios for this network. In the first scenario, due to high capacity requirements of V2I links, we formulate an optimization problem which maximizes the sumrate of two vehicles at all time slots satisfying the required rates of each vehicle at each time slot. This scenario is suitable for delay-tolerant cases, where for example both vehicles are downloading a video. We find optimal values for UAV trajectory, transmit powers of NOMA vehicles at the BS, and transmit power of the relay node to maximize this sum-rate. In the second scenario, in order to provide more uniform and fair rate performance between two users and among all time slots, we optimize the minimum rate of users at each time slot. This scenario is for delay-constrained applications such as safetycritical services. These optimization problems are non-convex and intractable to solve. In order to solve the above-mentioned non-convex problems, we apply the alternating optimization (AO) method. Hence, we divide our optimization problem into two separate sub-problems. In the first sub-problem, with a given trajectory planning, we optimize the transmit power of vehicles and the relay. With some manipulations, this problem is a difference of concave (DC) programming problem. In the second sub-problem, the trajectory of the UAV is optimized for given power allocations. Both of these sub-problems are still non-convex, and hence we apply the successive convex approximation (SCA) method to solve them.

Our system model can be generalized into a multi-user system with $K$ users. We can serve these disconnected vehicles by two methods. At the first method, we assign one UAV for each pair of NOMA users. Hence, we require $K / 2$ UAVs to serve $K$ users, and each of these UAVs must work in different sub-carriers. At the second method, we assume that we have only one UAV for all of the $K$ users. In order to have less decoding complexity, these $K$ users are divided into $K / 2$ groups with two users inside each group, and then NOMA scheme is applied for each pair. These pairs are distinguished by different sub-carriers. Note that in order to generalize our formulated optimization problems and their solutions for multi-user case, one can define user paring coefficients for NOMA scheme to pair vehicles. The problem of user pairing for NOMA scheme has been investigated well in the literature [33] and is beyond the scope of this paper.

The main contributions of this paper are summarized as follows:

- We show that in a two-user network with a dedicated AF relay, NOMA always has better or equal sum-rate performance in comparison to OMA at high SNR regime. Also, we show that OMA always has better min-rate performance than NOMA at high SNR regime.

- We show that for the cases where i) BS-to-relay link is weak, or ii) two users have similar links, or iii) BS-torelay link is similar to relay-to-weak user link, applying NOMA has negligible sum-rate gain in comparison to 
OMA. Hence, due to the complexity of SIC decoding at NOMA, we propose a dynamic NOMA/OMA scheme in which OMA is selected for transmission when applying NOMA has only negligible gain.

- We formulate two optimization problems in which the sum-rate and min-rate of two vehicles are maximized. The formulated problems are non-convex. Hence, using the $\mathrm{AO}$ method we divide the original problem into two separate sub-problems for optimizing trajectory and power allocations. These sub-problems are still non-convex, and hence we solve them via the SCA and DC methods. The proposed efficient method converges at few iterations.

\section{Organization}

The remainder of this paper is organized as follows. Section II presents the system model. Section III presents the proposed dynamic NOMA/OMA. Sections IV and V provide the formulated problems for the sum-rate and min-rate problems. Section VI provides simulation results to validate the performance of the proposed algorithms. Finally, Section VII concludes the paper.

\section{SYSTEM MODEL}

The considered system model consists of a BS, a UAVmounted relay, and two disconnected NOMA vehicles as depicted in Fig. 1 The vehicles receive different information (e.g., vehicle-specific control information) from the BS. We assume that the locations of vehicles are known a priory for the BS. Note that because vehicles are typically moving in a straight line at a street, this assumption is reasonable. Also, for some future applications, like autonomous driving, a central unit like a BS is responsible to control and drive vehicles, and hence it is aware of locations of vehicles. Due to high computational capabilities of BS rather than other nodes in our system model, we assume that the BS performs optimization algorithms. Due to existing obstacles, blocking buildings, and significant path loss, there is no direct link between the BS and the vehicles, and the UAV helps to extend coverage and establish a communication link for these disconnected vehicles. Note that we assume that vehicles can not establish communication link with other BSs, and therefore there is no inter-cell interference from other cells in our system model. We assume that the relay node applies the AF protocol and works in half-duplex mode. In AF protocol, the relay only amplifies and retransmits the received signal, and so the complexity of computations is reduced [34]. Note that in [4] the relay node buffers messages until a good communication link is established. The scheme in [4] adds an extra buffering delay to the system which is not desirable for ultra reliable low latency communication (URLLC). However, in this paper, we assume that at each time the relay node retransmits the received signal which imposes less delay in the transmission of data. All of the nodes are assumed to be equipped with a single antenna.

Hereafter, we show the BS, relay, vehicle 1, and vehicle 2 with subscripts $\mathrm{s}, \mathrm{r}, 1$, and 2, respectively. In our system, the BS is placed on the origin, and its coordinates is denoted

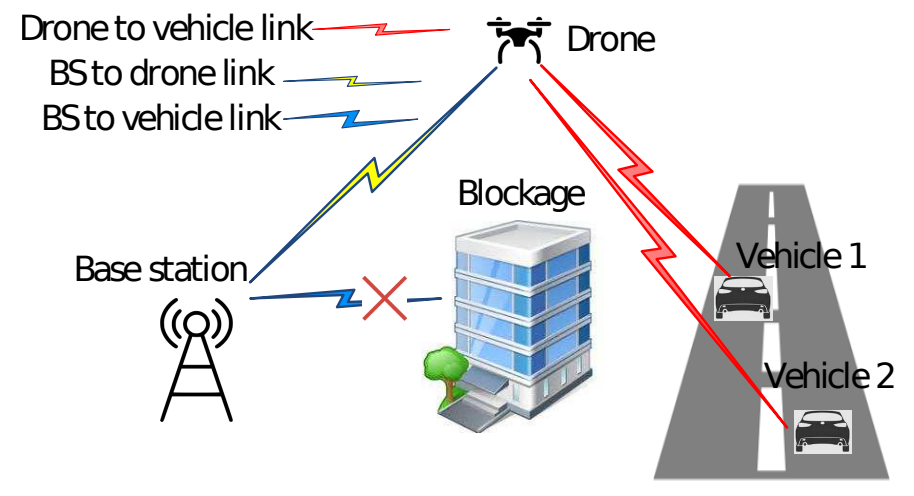

Fig. 1: System model for drone-assisted NR-V2X network.

by $\left(x_{B S}, y_{B S}, h_{B S}\right)=(0,0,0)$. Also, the coordinates of relay node, vehicle 1 , and vehicle 2 are denoted by $(x[n], y[n], h[n])$, $\left(x_{1}[n], y_{1}[n], 0\right)$ and $\left(x_{2}[n], y_{2}[n], 0\right)$, respectively, where $n$ indicates the number of time slots. Moreover, we assume that the UAV flies at a fixed height $h[n]=h$ [4]. We assume that there are predefined initial and final locations for the UAV flight path. Hence, the constraints $(x[0], y[0])=\left(x_{s}, y_{s}\right)$, and $(x[N+1], y[N+1])=\left(x_{f}, y_{f}\right)$ must be applied in the optimization problems where $N$ is the total number of time slots. We assume that the flight time $T$ for the UAV is divided into $N$ slots, such that $T=N \tau$, and $\tau$ is supposed to be small enough. Therefore, during each time slot, the position of the relay node is fixed [4]. In this paper, we assume that during flight time $T$, the UAV flies from position $\left(x_{s}, y_{s}, h\right)$ to position $\left(x_{f}, y_{f}, h\right)$. Also, the UAV can fly with the maximum speed denoted by $V$. Hence, during each time slot, the relay moves on the basis of the velocity constraint [4] as $(x[n]-x[n-1])^{2}+(y[n]-y[n-1])^{2} \leq(V \tau)^{2}, \quad \forall n$, which shows that due to the existence of a maximum velocity limitation, maximum displacement of the UAV must be less than $V \tau$ at each time slot $n$.

The channel power gains of the BS to the relay, the relay to vehicle 1 , and the relay to vehicle 2 are indicated by $h_{r}[n]$, $h_{1}[n]$, and $h_{2}[n]$, respectively. We assume that there is no small scale fading for the air-to-ground channels. We suppose that these channels are dominated by LOS links, and hence multipath fading can be ignored [4]. Hence, the channel power $h_{i}[n]$ follows the free space path loss model as

$$
h_{i}[n]=\beta_{0} d_{i}^{-2}[n]=\frac{\beta_{0}}{\left(x[n]-x_{i}[n]\right)^{2}+\left(y[n]-y_{i}[n]\right)^{2}+h^{2}},
$$

for $n=1, \ldots, N, i=1,2$, where $\beta_{0}$ denotes the channel power at the reference distance $d_{0}=1 \mathrm{~m}$. We consider independent additive white gaussian noise (AWGN) $z_{k}[n]$ with the distribution $C N\left(0, \sigma_{k}^{2}[n]\right)(k \in\{r, 1,2\}, \forall n)$ in which $\sigma_{k}^{2}[n]=\sigma^{2}$ shows the variance of the noise for node $k$ at time slot $n$ 1 $\bar{P}_{s}$ and $\bar{P}_{r}$ indicate the average transmit power at each

\footnotetext{
${ }^{1}$ Note that for thermal noise at receiver we have $N_{0}=K T$, in which $K$ is Boltzmann's constant, and $T$ is the receiver system temperature in kelvins. Because the UAV flies at the height of $h=100 \mathrm{~m}$, small changes of receiver's temperature between $h=0 \mathrm{~m}$ and $h=100 \mathrm{~m}$ have a small impact on $N_{0}$. Hence, we assume same $N_{0}$ for aerial and terrestrial nodes.
} 
time slot at the source and relay nodes, respectively. During $N$ time slots, the total transmit energy for the source and relay nodes must be less than $E_{s}=N \bar{P}_{s}$ and $E_{r}=N \bar{P}_{r}$, respectively [4]. Hence, the BS and UAV can consume different powers at different time slots conditioned upon their energy consumption constraint. Finally, we assume that the relay node operates in frequency division duplexing (FDD) mode with equal bandwidth allocated for information reception from the BS and transmission to the vehicles.

\section{DYNAMIC NOMA/OMA SCHEME}

In the NOMA scheme, the BS sends the combination of vehicle messages to the relay node based on superposition coding (SC) as $s_{3}[n]=\sqrt{p_{1}[n]} s_{1}[n]+\sqrt{p_{2}[n]} s_{2}[n]$, where $p_{1}[n]$ and $p_{2}[n]$ are power allocation coefficients at time slot $n$ for each vehicle, and we call them NOMA coefficients. $s_{1}[n]$ and $s_{2}[n]$ are the messages of vehicle 1 and vehicle 2 , respectively. We assume that $E\left\{\left|s_{1}[n]\right|^{2}\right\}=E\left\{\left|s_{2}[n]\right|^{2}\right\}=1$. In NOMA, less power is allocated to the strong vehicle, and more power is allocated to the weak vehicle [12]. Then we can perform SIC to remove far vehicle interference from the received signal at the strong vehicle. Note that due to assuming the free space path loss model for air to ground channels, we only need to sort NOMA vehicles based on their distances. As we can see in Fig. 1, based on the trajectory of the UAV and the locations of vehicles, vehicle 1 (vehicle 2) can be near vehicle in some time slots. Hence, when vehicle 1 (vehicle 2 ) is the near vehicle, we apply SIC at vehicle 1 (vehicle 2 ) to remove the interference of vehicle 2 (vehicle 1 ). The received signal at the relay node at time slot $n$ is given by $y_{r}[n]=\sqrt{h_{r}[n] p_{1}[n]} s_{1}[n]+\sqrt{h_{r}[n] p_{2}[n]} s_{2}[n]+z_{r}[n]$. The relay works in the AF mode in our system and amplifies the received signal at each time slot with amplification gain $\rho[n]$. According to [34], and assuming that we allocate power $p_{r}[n]$ for the UAV, we can write the amplification gain as $\rho[n]=\frac{p_{r}[n]}{\left(p_{1}[n]+p_{2}[n]\right) h_{r}[n]+\sigma^{2}}$. Then, the relay transmits the amplified signal $\sqrt{\rho[n]} y_{r}[n]$ to the vehicles. The received signal at each vehicle is given by

$$
\begin{aligned}
y_{k}[n] & =\sqrt{\rho[n] h_{r k}[n]} y_{r}[n]+z_{k}[n] \\
& =\sqrt{\rho[n] h_{r k}[n] h_{r}[n] p_{1}[n]} s_{1}[n] \\
& +\sqrt{\rho[n] h_{r k}[n] h_{r}[n] p_{2}[n]} s_{2}[n] \\
& +\sqrt{\rho[n] h_{r k}[n]} z_{r}[n]+z_{k}[n], \quad k=1,2 .
\end{aligned}
$$

One can see from (2) that for the case where vehicle 1 (vehicle 2) is the near vehicle, in order to perform SIC, we have to allocate more power for vehicle 2 (vehicle 1 ). Note that due to the mobility of the vehicles and the aerial relay in our system, the channel power gain between nodes changes a lot. Hence, in contrast to the scenarios with fixed users in which the role of the strong user is given to the user closest to the $\mathrm{BS}$, the roles of strong and weak users alternate between the two vehicles in different time slots for our system. This means that the vehicle which must perform SIC alternates in different time slots, and consequently, the complexity of decoding is divided between vehicles. Also, we will prove that in some cases NOMA is not superior to OMA. Applying OMA in

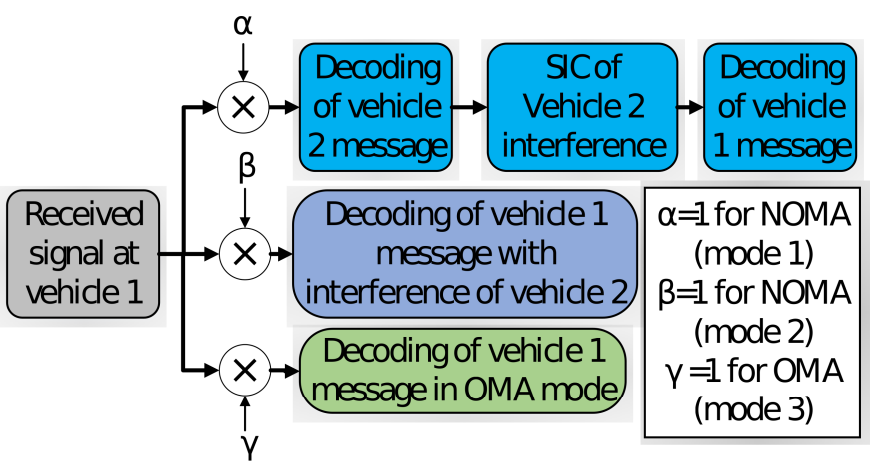

Fig. 2: Decoding structure at vehicle 1's receiver with proposed dynamic NOMA/OMA.

these cases reduces the complexity of performing SIC at the vehicles in the cost of a minor decrease in throughput. Based on these observations, we propose a dynamic NOMA/OMA scheme with the following three modes for transmission of vehicle messages: SIC at vehicle 1, SIC at vehicle 2, and OMA which are indicated by mode $1(m=1)$, mode $2(m=2)$, and mode $3(m=3)$, respectively. The structure of the receiver's decoder at vehicle 1 for the proposed dynamic NOMA/OMA scheme has been shown in Fig. 2. Note that the same structure can be depicted for the decoder of vehicle 2 .

\section{A. Mode 1: SIC at Vehicle 1}

In this case, vehicle 1 is the near vehicle and performs SIC to remove the interference of vehicle 2 . Hereafter, we name the multiple access scheme of this case 'mode 1' ( $m=1)$. At vehicle 1 , message of vehicle 2 is decoded first. Then, this decoded message is subtracted from the received signal at vehicle 1 . Finally, vehicle 1 decodes its own message without any interference. Hence, the effective SINR of the vehicle 2 message observed at vehicle 1 equals $\gamma_{1,2}[n]=\frac{\rho[n] h_{1}[n] h_{r}[n] p_{2}[n]}{\rho[n] h_{1}[n] h_{r}[n] p_{1}[n]+\rho[n] h_{1}[n] \sigma^{2}+\sigma^{2}}$, and the achievable rate of decoding the vehicle 2 message at vehicle 1 equals $R_{1,2}[n]=\log _{2}\left(1+\gamma_{1,2}[n]\right)$. Then, the achievable rate of vehicle 1 equals

$$
\begin{aligned}
R_{1}^{1}[n] & =\log _{2}(1+ \\
& \left.\frac{p_{r}[n] h_{1}[n] h_{r}[n] p_{1}[n]}{p_{r}[n] h_{1}[n] \sigma^{2}+\left(p_{1}[n]+p_{2}[n]\right) h_{r}[n] \sigma^{2}+\sigma^{4}}\right),
\end{aligned}
$$

in which $R_{k}^{m}[n]$ indicates the achievable rate of vehicle $k$ at mode $m$. From (2), the achievable rate of the vehicle 2 message at vehicle 2 equals $R_{2,2}[n]=\log _{2}(1+$

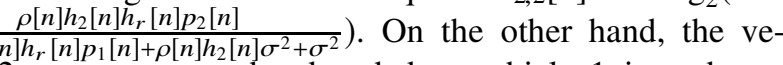
$\rho[n] h_{2}[n] h_{r}[n] p_{1}[n]+\rho[n] h_{2}[n] \sigma^{2}+\sigma^{2}$
hicle 2 message must be decoded at vehicle 1 in order to perform SIC. Hence, the achievable rate of vehicle 2 equals $R_{2}[n]=\min \left(R_{1,2}[n], R_{2,2}[n]\right)$. In this mode, we suppose that vehicle 1 is closer to the UAV in comparison to vehicle 2 . Hence, we write $h_{1}[n]>h_{2}[n]$. Therefore, $R_{1,2}[n]>R_{2,2}[n]$, and we can write (4) at the top of the next page.

\section{B. Mode 2: SIC at Vehicle 2}

In this mode, vehicle 2 is the near vehicle, and performs SIC to remove the interference of vehicle 1 . The formulation 


$$
R_{2}^{1}[n]=\log _{2}\left(1+\frac{p_{r}[n] h_{2}[n] h_{r}[n] p_{2}[n]}{p_{r}[n] h_{2}[n] h_{r}[n] p_{1}[n]+p_{r}[n] h_{2}[n] \sigma^{2}+\left(p_{1}[n]+p_{2}[n]\right) h_{r}[n] \sigma^{2}+\sigma^{4}}\right) .
$$

of achievable rates for both vehicles in this mode is similar to the previous case except that we perform SIC at vehicle 2 . Hence, we only write the achievable rates of vehicles in this section. The achievable rate of vehicle 2 and vehicle 1 are given by

$$
\begin{aligned}
& R_{2}^{2}[n]=\log _{2}(1+ \\
& \left.\quad \frac{p_{r}[n] h_{2}[n] h_{r}[n] p_{2}[n]}{p_{r}[n] h_{2}[n] \sigma^{2}+\left(p_{1}[n]+p_{2}[n]\right) h_{r}[n] \sigma^{2}+\sigma^{4}}\right),
\end{aligned}
$$

and (6) at the top of the next page.

\section{Mode 3: OMA Scheme}

We apply frequency division multiple access (FDMA) for two vehicles in this mode. We assume that the bandwidth of each time slot $n$ is equally divided between two vehicles at both the BS and relay nodes and that the vehicles send their messages in an orthogonal manner. Hereafter, we name this case 'mode 3' $(\mathrm{m}=3)$. We assume that different transmit powers $p_{1}[n]$ and $p_{2}[n]$ at the $\mathrm{BS}$ are allocated to vehicle 1 and vehicle 2 , respectively. The BS sends $\sqrt{p_{1}[n]} s_{1}[n]$, and $\sqrt{p_{2}[n]} s_{2}[n]$, at two orthogonal frequency bands of each time slot. The received signals at the relay node equals $y_{r}^{k}[n]=\sqrt{h_{r}[n] p_{k}[n]} s_{k}[n]+z_{r}[n]$ for $k=1,2$. Note that due to dividing frequency bands for the two vehicles, the variance of the noise $z_{r}[n]$ is half of the NOMA case. Hence, we assume that this noise has the same variance $\sigma_{O}^{2}=0.5 \sigma^{2}$ in all time slots. At the relay node, we assume that the power of relay $p_{r}[n]$ is equally divided between two vehicles. Therefore, we can write the amplification gain of each vehicle message as $\rho_{k}[n]=\frac{0.5 p_{r}[n]}{p_{k}[n] h_{r}[n]+\sigma_{O}^{2}}$ for $k=1,2$. Then, the relay transmits the amplified signal $\sqrt{\rho_{k}[n]} y_{r}^{k}[n]$ to the vehicles. The received signal at each vehicle is given by

$$
\begin{aligned}
y_{k}[n] & =\sqrt{\rho_{k}[n] h_{k}[n]} y_{r}^{k}[n]+z_{k}[n] \\
& =\sqrt{\rho_{k}[n] h_{k}[n]}\left(\sqrt{h_{r}[n] p_{k}[n]} s_{k}[n]+z_{r}[n]\right)+z_{k}[n] \\
& =\sqrt{\rho_{k}[n] h_{k}[n] h_{r}[n] p_{k}[n]} s_{k}[n] \\
& +\sqrt{\rho_{k}[n] h_{k}[n]} z_{r}[n]+z_{k}[n], \quad k=1,2 .
\end{aligned}
$$

We assume that $z_{k}[n]$ has the same variance $\sigma_{O}^{2}=0.5 \sigma^{2}$ at each vehicle. From (7), the effective SINR of each vehicle equals $\gamma_{k}[n]=\frac{\rho_{k}[n] h_{k}[n] h_{r}[n] p_{k}[n]}{\rho_{k}[n] h_{k}[n] \sigma_{O}^{2}+\sigma_{O}^{2}}$, and the rates of vehicles are given by

$$
R_{k}^{3}[n]=\frac{1}{2} \log _{2}\left(1+\frac{p_{r}[n] h_{k}[n] h_{r}[n] p_{k}[n]}{p_{r}[n] h_{k}[n] \sigma_{O}^{2}+2 p_{k}[n] h_{r}[n] \sigma_{O}^{2}+2 \sigma_{O}^{4}}\right),
$$

for $k=1,2$.

\section{Proposed Dynamic NOMA/OMA Scheme}

In this section, we first introduce two propositions which compare the sum-rate and min-rate performance of NOMA and OMA at the high SNR regime. We assume that $R_{i, j}^{\infty}$ shows the approximated value for rate at the high SNR regime, where $i \in\{S, M\}$ differentiates sum-rate and min-rate, and $j \in\{O, N\}$ differentiates OMA and NOMA schemes.

Proposition 1. In a two-user network with a dedicated $A F$ relay and in the absence of a direct link between the $B S$ and two users, NOMA always has better or equal sum-rate performance in comparison to OMA at high SNR regime.

Proof. We use the same notations as in the system model section except that we take the transmit SNRs at the BS and relay nodes (i.e., $\rho=\frac{\bar{P}_{s}}{\sigma^{2}}=\frac{\bar{P}_{r}}{\sigma^{2}}=\frac{P}{\sigma^{2}}$ ) into channel power gains. Hence, new channel power gains are $h_{i}^{\infty}=\rho h_{i}$ $(i \in\{r, 1,2\})$ where the superscript $\infty$ indicates the high SNR regime. Also, with this new notation for channel power gain, we have $p_{1}+p_{2}=1$ and $p_{r}=1$. Assuming that $h_{1}>h_{2}$, we have to perform SIC at user 1. Hence, utilizing the achievable rates of users at mode 1 from (3) and (4) we have

$$
\begin{aligned}
R_{S, N}^{\infty} & =R_{1}^{1, \infty}+R_{2}^{1, \infty}=\log _{2}\left(1+\frac{p_{r} h_{1}^{\infty} h_{r}^{\infty} p_{1}}{p_{r} h_{1}^{\infty}+\left(p_{1}+p_{2}\right) h_{r}^{\infty}+1}\right) \\
& +\log _{2}\left(1+\frac{p_{r} h_{2}^{\infty} h_{r}^{\infty} p_{2}}{p_{r} h_{2}^{\infty} h_{r}^{\infty} p_{1}+p_{r} h_{2}^{\infty}+\left(p_{1}+p_{2}\right) h_{r}^{\infty}+1}\right) \\
& \approx \log _{2}\left(\frac{h_{1}^{\infty} h_{r}^{\infty}}{h_{1}^{\infty}+h_{r}^{\infty}}\right)+\log _{2}\left(p_{1}\right)+\log _{2}\left(1+\frac{p_{2}}{p_{1}}\right) \\
& =\log _{2}\left(\frac{h_{1}^{\infty} h_{r}^{\infty}}{h_{1}^{\infty}+h_{r}^{\infty}}\right) \approx \log _{2}\left(\min \left(h_{r}^{\infty}, h_{1}^{\infty}\right)\right) .
\end{aligned}
$$

On the other hand, for the OMA scheme, we use the rates of the users at mode 3 from (8) as

$$
\begin{aligned}
R_{S, O}^{\infty} & =\sum_{k=1}^{2} R_{k}^{3, \infty}=\sum_{k=1}^{2} \frac{1}{2} \log _{2}\left(1+\frac{2 p_{r} h_{k}^{\infty} h_{r}^{\infty} p_{k}}{p_{r} h_{k}^{\infty}+2 p_{k} h_{r}^{\infty}+1}\right) \\
& \approx \sum_{k=1}^{2} \frac{1}{2} \log _{2}\left(\min \left(h_{r}^{\infty}, h_{k}^{\infty}\right)\right),
\end{aligned}
$$

where we considered $p_{1}=p_{2}=0.5$ in (10). We can see from (9) and (10) that with $h_{1}^{\infty}>h_{2}^{\infty}$, NOMA always has equal or better sum-rate performance in comparison to the OMA scheme. Actually, based on the channel power gains of links, three cases may occur for sum-rate:

Case 1: When we have $h_{r}^{\infty}>h_{1}^{\infty}>h_{2}^{\infty}$ (Fig. 3a), the sum-rates of NOMA and OMA are $\log _{2}\left(h_{1}^{\infty}\right)$ and $\frac{1}{2} \log _{2}\left(h_{1}^{\infty}\right)+\frac{1}{2} \log _{2}\left(h_{2}^{\infty}\right)$, respectively. NOMA has better performance than OMA in this case as $R_{S, N}^{\infty}-R_{S, O}^{\infty} \approx \log _{2}\left(h_{1}^{\infty}\right)-\frac{1}{2} \log _{2}\left(h_{1}^{\infty}\right)-\frac{1}{2} \log _{2}\left(h_{2}^{\infty}\right)=$ $\frac{1}{2} \log _{2}\left(\frac{h_{1}^{\infty}}{h_{2}^{\infty}}\right)$. Hence, by increasing the difference between $h_{1}^{\infty}$ and $h_{2}^{\infty}$, the superiority of NOMA increases.

Case 2: When we have $h_{1}^{\infty}>h_{r}^{\infty}>h_{2}^{\infty}$ (Fig. 3b), the sum-rates 


$$
R_{1}^{2}[n]=\log _{2}\left(1+\frac{p_{r}[n] h_{1}[n] h_{r}[n] p_{1}[n]}{p_{r}[n] h_{1}[n] h_{r}[n] p_{2}[n]+p_{r}[n] h_{1}[n] \sigma^{2}+\left(p_{1}[n]+p_{2}[n]\right) h_{r}[n] \sigma^{2}+\sigma^{4}}\right)
$$

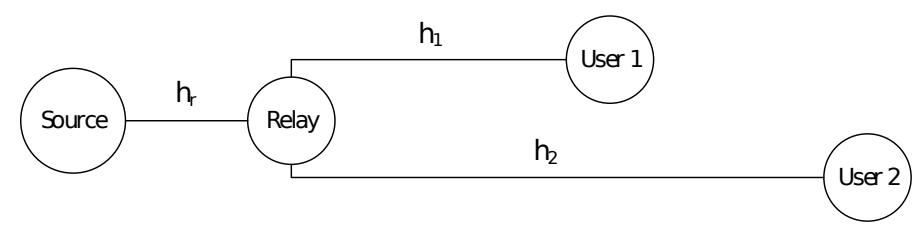

(a) Case 1: $h_{r}>h_{1}>h_{2}$

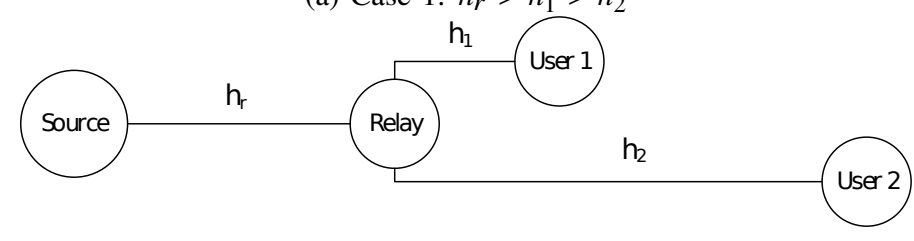

(b) Case 2: $h_{1}>h_{r}>h_{2}$

Fig. 3: Two cases where NOMA has better sum-rate than OMA (refer to Proposition 11).

of NOMA and OMA are $\log _{2}\left(h_{r}^{\infty}\right)$ and $\frac{1}{2} \log _{2}\left(h_{r}^{\infty}\right)+\frac{1}{2} \log _{2}\left(h_{2}^{\infty}\right)$, respectively. One can see that NOMA has better performance than OMA as $R_{S, N}^{\infty}-R_{S, O}^{\infty} \approx \log _{2}\left(h_{r}^{\infty}\right)-\frac{1}{2} \log _{2}\left(h_{r}^{\infty}\right)-$ $\frac{1}{2} \log _{2}\left(h_{2}^{\infty}\right)=\frac{1}{2} \log _{2}\left(\frac{h_{r}^{\infty}}{h_{2}^{\infty}}\right)$. We see that by increasing the difference between $h_{r}^{\infty}$ and $h_{2}^{\infty}$, the superiority of NOMA increases.

Case 3: When we have $h_{1}^{\infty}>h_{2}^{\infty}>h_{r}^{\infty}$, the sum-rates of both schemes equals $\log _{2}\left(h_{2}^{\infty}\right)$. Hence, NOMA has the same performance as OMA for the case where the BS-to-relay link is worse than the relay-to-user links. Consequently, the proof is completed.

Remark 1. Proposition 1 proves that, for the cases where two users have similar links, or where BS-to-relay link is similar with relay-to-weak user link, applying NOMA has negligible sum-rate gain. Also, when the BS-to-relay link is weak, NOMA and OMA have similar sum-rate.

Proposition 2. In a two-user network with a dedicated $A F$ relay and in the absence of a direct link between the $B S$ and two users, OMA has superior minimum-rate performance in comparison to NOMA at the high SNR regime.

Proof. With the same notations used in the proof of Proposition 1 the minimum achievable rates of users at high SNRs in mode 1 can be written from (3), (4) and (9) as

$$
\begin{aligned}
R_{M, N}^{\infty} & =\min \left(R_{1}^{1, \infty}, R_{2}^{1, \infty}\right) \\
& \approx \min \left(\log _{2}\left(\frac{h_{1}^{\infty} h_{r}^{\infty}}{h_{1}^{\infty}+h_{r}^{\infty}}\right), \log _{2}\left(\frac{p_{2}}{p_{1}}\right)\right)=\log _{2}\left(\frac{p_{2}}{p_{1}}\right) .
\end{aligned}
$$

For the OMA scheme we use the rates of the users at mode 3 from (8) and (10) as

$$
R_{M, O}^{\infty}=\min \left(R_{1}^{3, \infty}, R_{2}^{3, \infty}\right)=R_{2}^{3, \infty} \approx \frac{1}{2} \log _{2}\left(\frac{h_{2}^{\infty} h_{r}^{\infty}}{h_{2}^{\infty}+h_{r}^{\infty}}\right)
$$

We can see from (11) and (12) that the OMA scheme always has better min-rate performance in comparison to the NOMA

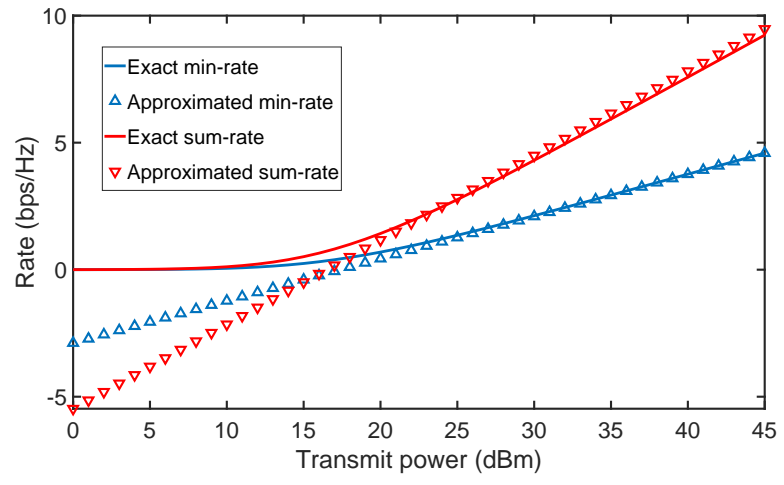

(a) OMA scheme.

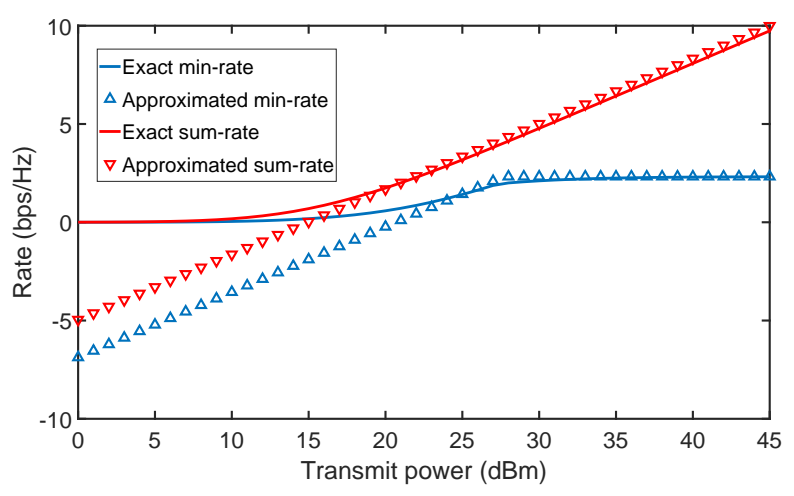

(b) NOMA scheme.

Fig. 4: Approximated and exact rates for sum-rate and min-rate of OMA and NOMA schemes at Proposition 1 and Proposition 2

scheme at the high SNR regime. Hence, the proof is completed.

Remark 2. Note that the results in Propositions $\square$ and 2 are not limited to an aerial AF relay; rather, they are general results for any two-user network with a dedicated $A F$ relay.

In Fig. 4, we can see the approximated and exact rates for sum-rate and min-rate of OMA and NOMA schemes at Proposition 11 and Proposition 2 In Fig. 4a we can see that when $P>22 \mathrm{dBm}$, we have $\left|R_{S, O}^{\infty}-R_{S, O}\right| \leq 0.2$ and $\left|R_{M, O}^{\infty}-R_{M, O}\right| \leq 0.2$ for the approximations (10) and (12) at sum-rate and min-rate of OMA scheme, respectively. Also, we can see in Fig. $4 \mathrm{~b}$ that when $P>22 \mathrm{dBm}$, we have $\left|R_{S, N}^{\infty}-R_{S, N}\right| \leq 0.2$ and $\left|R_{M, N}^{\infty}-R_{M, N}\right| \leq 0.2$ for the approximations (9) and (11) at sum-rate and min-rate of NOMA scheme, respectively. Note that for Fig. 4, we have considered default simulation parameters as in Section VI except that $\left(x_{s}, y_{s}\right)=(250,250)$ and $\left(y_{1}, y_{2}\right)=(400,500)$.

NOMA scheme requires more complex receiver rather than OMA (Fig. 2). Actually, performing SIC at near user leads to a more complex decoder at the receiver of near user 
TABLE I: Proposed dynamic NOMA/OMA scheme.

\begin{tabular}{|c|c|c|c|c|c|c|}
\hline State & Channel gain order & Channel difference & Multiple access scheme & $\alpha_{S, n}$ & $\beta_{S, n}$ & $\gamma_{s, n}$ \\
\hline $\mathrm{s}=1$ & \multirow[t]{2}{*}{$h_{r}>h_{1}>h_{2}$} & $\frac{1}{2} \log _{2}\left(\frac{h_{1}}{h_{2}}\right)>R_{\mathrm{th}}^{S}$ & NOMA-SIC at vehicle $1(\mathrm{~m}=1)$ & 1 & 0 & 0 \\
\hline $\mathrm{s}=2$ & & $\frac{1}{2} \log _{2}\left(\frac{h_{1}}{h_{2}}\right)<R_{\mathrm{th}}^{S}$ & OMA $(m=3)$ & 0 & 0 & 1 \\
\hline $\mathrm{s}=3$ & \multirow[t]{2}{*}{$h_{1}>h_{r}>h_{2}$} & $\frac{1}{2} \log _{2}\left(\frac{h_{r}}{h_{2}}\right)>R_{\mathrm{th}}^{S}$ & NOMA- SIC at vehicle $1(\mathrm{~m}=1)$ & 1 & 0 & 0 \\
\hline $\mathrm{s}=4$ & & $\frac{1}{2} \log _{2}\left(\frac{h_{r}}{h_{2}}\right)<R_{\mathrm{th}}^{S}$ & OMA $(\mathrm{m}=3)$ & 0 & 0 & 1 \\
\hline $\mathrm{s}=5$ & $h_{1}>h_{2}>h_{r}$ & - & OMA $(\mathrm{m}=3)$ & 0 & 0 & 1 \\
\hline $\mathrm{s}=6$ & \multirow{2}{*}{$h_{r}>h_{2}>h_{1}$} & $\frac{1}{2} \log _{2}\left(\frac{h_{2}}{h_{1}}\right)>R_{\mathrm{th}}^{S}$ & NOMA- SIC at vehicle $2(\mathrm{~m}=2)$ & 0 & 1 & 0 \\
\hline $\mathrm{s}=7$ & & $\frac{1}{2} \log _{2}\left(\frac{h_{2}}{h_{1}}\right)<R_{\mathrm{th}}^{S}$ & OMA $(\mathrm{m}=3)$ & 0 & 0 & 1 \\
\hline $\mathrm{s}=8$ & \multirow{2}{*}{$h_{2}>h_{r}>h_{1}$} & $\frac{1}{2} \log _{2}\left(\frac{h_{r}}{h_{1}}\right)>R_{\mathrm{th}}^{S}$ & NOMA- SIC at vehicle $2(\mathrm{~m}=2)$ & 0 & 1 & 0 \\
\hline $\mathrm{s}=9$ & & $\frac{1}{2} \log _{2}\left(\frac{h_{r}}{h_{1}}\right)<R_{\mathrm{th}}^{S}$ & OMA $(m=3)$ & 0 & 0 & 1 \\
\hline$s=10$ & $h_{2}>h_{1}>h_{r}$ & & OMA $(\mathrm{m}=3)$ & 0 & 0 & 1 \\
\hline
\end{tabular}

[35], and consequently, more required power. Hence, more power consumption and complexity are the costs that we pay at receiver side to have better throughput by applying the NOMA scheme. Based on the observations in Proposition 1 and Remark 1 we propose a dynamic NOMA/OMA scheme in which OMA is selected when applying NOMA has zero or only negligible gain. According to Proposition 1, the gain of NOMA scheme over OMA relies on the channel gains of BSto-relay and relay-to-users links. Due to movements of UAV node and vehicles in our system model, these channel gains are changing fast, and hence, their orders change quickly. Hence, as one can see in Fig. 2, transceiver parts of nodes have to quickly switch among three working modes, i.e., OMA, NOMA with SIC at user 1 and NOMA with SIC at user 2. These quick switches causes more power and complexity costs for transceiver of BS, relay and users. In order to prevent our dynamic scheme from the quick switches among working modes and keep the complexity costs of applying NOMA scheme as little as possible, we define a superiority threshold parameter $R_{t h}^{S}$. When the sum-rate superiority of NOMA in comparison to OMA is less than this threshold value, i.e., $R_{S, N}-R_{S, O}<R_{\mathrm{th}}^{S}$, our dynamic scheme is set to OMA mode. $R_{t h}^{S}$ can be chosen based on the priorities of the network operator between complexity and throughput. It is clear that increasing $R_{t h}^{S}$ leads to selection of more OMA working modes by proposed dynamic scheme which leads to less decoding and switching complexity in transceiver design in the cost of missing more throughput.

Note that in Proposition 1 we assumed that $h_{1}>h_{2}$, and we have three other states for the channel gain orders in the case where $h_{2}>h_{1}$. At each state, based on the order and difference of channel gains, we can apply NOMA or OMA. At time slot $n$ and state $s$, in order to determine three modes for multiple access scheme, i.e., $m=1,2,3$, we introduce three binary matrices, namely $\mathbf{A}=\left[\alpha_{s, n}\right]_{S \times N}, \mathbf{B}=\left[\beta_{s, n}\right]_{S \times N}$, and $\boldsymbol{\Gamma}=\left[\gamma_{s, n}\right]_{S \times N}$, respectively. The summary of the proposed dynamic multiple access scheme is shown in Table []

\section{Sum-Rate Maximization}

In this section, we formulate the optimization problem of our system. We aim to optimize the end-to-end sumrate by finding the optimal values for relay trajectory $(\mathbf{X}=$ $\left[x_{r}[1], \ldots, x_{r}[N]\right]$ and $\left.\mathbf{Y}=\left[y_{r}[1], \ldots, y_{r}[N]\right]\right)$, power coefficients of NOMA vehicles at the $\mathrm{BS}\left(\mathbf{P}_{\mathbf{1}}=\left[p_{1}[1], \ldots, p_{1}[N]\right]\right.$ and $\left.\mathbf{P}_{2}=\left[p_{2}[1], \ldots, p_{2}[N]\right]\right)$, allocated power of the relay node $\left(\mathbf{P}_{\mathbf{r}}=\left[p_{r}[1], \ldots, p_{r}[N]\right]\right)$, and operation mode selection matrices for multiple access scheme $\left(\mathbf{A}=\left[\alpha_{s, n}\right]_{S \times N}, \mathbf{B}=\right.$ $\left[\beta_{S, n}\right]_{S \times N}$, and $\left.\boldsymbol{\Gamma}=\left[\gamma_{s, n}\right]_{S \times N}\right)$. Therefore, the optimization problem can be formulated as

$$
\begin{aligned}
& \text { (P1): } \max _{\mathbf{P}_{1}, \mathbf{P}_{2}, \mathbf{P}_{r}, \mathbf{X}, \mathbf{Y}, \mathbf{A}, \mathbf{B}, \boldsymbol{\Gamma}} \sum_{n=1}^{N} \sum_{s=1}^{S} \sum_{k=1}^{K} \alpha_{s, n} R_{k}^{1}[n] \\
& +\beta_{s, n} R_{k}^{2}[n]+\gamma_{s, n} R_{k}^{3}[n] \\
& \text { s.t. } \quad \sum_{s=1}^{S} \alpha_{s, n} R_{k}^{1}[n]+\beta_{s, n} R_{k}^{2}[n]+\gamma_{s, n} R_{k}^{3}[n] \geq R_{k}^{t}, \quad \forall n, \forall k, \\
& (x[n]-x[n-1])^{2}+(y[n]-y[n-1])^{2} \leq(V \tau)^{2}, \\
& n=2, \ldots, N \text {, } \\
& \left(x[1]-x_{S}\right)^{2}+\left(y[1]-y_{s}\right)^{2} \leq(V \tau)^{2}, \\
& \left(x[N]-x_{f}\right)^{2}+\left(y[N]-y_{f}\right)^{2} \leq(V \tau)^{2}, \\
& x_{\min } \leq x[n] \leq x_{\max }, y_{\min } \leq y[n] \leq y_{\max }, \forall n \text {, } \\
& \sum_{n=1}^{N} \sum_{s=1}^{S}\left(\alpha_{s, n}+\beta_{s, n}+\gamma_{s, n}\right)\left(p_{1}[n]+p_{2}[n]\right) \leq E_{S} \text {, } \\
& \sum_{n=1}^{N} p_{r}[n] \leq E_{r}, \\
& \sum_{s=1}^{S} \alpha_{s, n}\left(p_{2}[n]-p_{1}[n]\right)+\beta_{s, n}\left(p_{1}[n]-p_{2}[n]\right) \geq 0, \quad \forall n, \\
& p_{r}[n] \geq 0, p_{1}[n] \geq 0, \quad p_{2}[n] \geq 0, \quad \forall n,
\end{aligned}
$$

where $R_{1}^{1}, R_{2}^{1}, R_{1}^{2}, R_{2}^{2}$, and $R_{k}^{3}(k \in\{1,2\})$ was calculated in (3), (4), (6), (5), and (8), respectively. Note that $R_{k}^{m}$ indicates the rate of vehicle $k$ at mode $m$, and $K=2$ is the number of vehicles. Constraint (14) guarantees minimum target rate $R_{k}^{t}$ for vehicle $k$ at time slot $n$. Constraint (15) indicates the velocity constraint for the movements of the relay node at each time slot. Constraints (16) and (17) show that the UAV must start and finish its trajectory at specified locations. Constraint (18) indicates the range that the UAV can fly. Constraints (19) and (20) indicate the total energy constraints for the BS and relay nodes, respectively, in which $E_{s}=N \bar{P}_{s}$ and $E_{r}=N \bar{P}_{r}$. Constraint (21) imposes a constraint to the allocated powers of the vehicles, in order to perform SIC at each time slot $n$. Note that $\alpha_{s, n}, \beta_{s, n}$, and $\gamma_{s, n}$ are indicator functions for three operation modes, and at each time slot $n$, only one of them must equal one.

The objective function of (P1) and constraint (14) are not concave with respect to trajectory and power variables. Also, (P1) contains operation mode coefficients which are integer variables. Hence, (P1) is a NP-hard non-convex optimization problem and can not be solved using standard convex optimization methods. In order to solve this problem, we use the AO method. Therefore, we first solve trajectory planning and operation mode selection sub-problem with fixed power allocations. Then, we propose a solution for power allocation sub-problem with fixed trajectory and operation mode coefficients. Finally, by alternatively optimizing the trajectory, mode 
selection, and power allocation sub-problems, we propose an iterative algorithm to solve (P1).

\section{A. Trajectory Planning and Operation Mode Selection for Fixed Power Allocation}

In this subsection, we solve the first sub-problem of (P1) to find the optimal values for the UAV trajectory (i.e., $\mathbf{X}$ and $\mathbf{Y}$ ) and operation mode coefficients (i.e., $\mathbf{A}, \mathbf{B}$, and $\mathbf{\Gamma}$ ) assuming that the allocated powers are given. Hence, we have

$$
\begin{array}{ll}
\text { (P1.1): } \max _{\mathbf{X}, \mathbf{Y}, \mathbf{A}, \mathbf{B}, \boldsymbol{\Gamma}} & \sum_{n=1}^{N} \sum_{s=1}^{S} \sum_{k=1}^{K} \alpha_{s, n} R_{k}^{1}[n] \\
& +\beta_{s, n} R_{k}^{2}[n]+\gamma_{s, n} R_{k}^{3}[n] \\
\text { s.t. } & (14),(15),(16),(17),(18) .
\end{array}
$$

If we replace the channel model formula in (1) at each vehicle rate formula in the equations (3), (4), (5), (6) and (8), one can see that $(\mathrm{P} 1.1)$ is not a convex problem with respect to variables $\mathbf{X}$ and $\mathbf{Y}$. Indeed, the objective function in (23) and constraint (14) are not concave functions. Also, note that operation mode coefficients are integer variables. We propose to use the AO method for solving this problem. For fixed trajectory planning, the channel power gains are known and we can use Table I to determine $\mathbf{A}, \mathbf{B}$, and $\boldsymbol{\Gamma}$. For given operation mode coefficients, (P1.1) is still non-convex, and we utilize the SCA method in which a concave approximation of the original objective function is maximized iteratively.

Lemma 1. Consider $x$ and $y$ as two variables, and $a, b$, $c$, and $d$ as constants. The function $f(x, y)=\frac{1}{a x+b y+c x y+d}$, is a convex function with respect to $x$ and $y$, if and only if $a x+b y+c x y+d>0$, and $a b=c d$.

Proof. See Appendix A

In the following proposition, we use Lemma 1 to find a concave approximation for the rates. Note that the location of the UAV is indicated by $\left(x^{l}[n], y^{l}[n]\right)$ at the $l^{\text {th }}$ iteration. Also, the lower-bound (LB) approximated rate and SINR of vehicle $k(k=1,2)$ in mode $m(m=1,2,3)$ and at iteration $l$ is indicated by $R_{k, \mathrm{lb}}^{l, m}[n]$ and $\gamma_{k, \mathrm{lb}}^{l, m}[n]$, respectively. Note that we remove the time slot number $n$ in the following proposition equations to make the formulas simpler to read.

Proposition 3. At each time slot $n$, for any given power allocation at the BS and UAV nodes (i.e., $p_{r}, p_{1}$, and $p_{2}$ ), and for any trajectory at the $l^{\text {th }}$ iteration (i.e., $x^{l}$, and $y^{l}$ ), one approximated concave non-decreasing lower-bound of the achievable rate of vehicle $k$ at mode $m$ and the $(l+1)^{\text {th }}$ iteration of the SCA method is

$$
\begin{aligned}
R_{k, l b}^{l+1, m} & =c_{m} \log _{2}\left(1+\gamma_{k, l b}^{l, m}+d_{k, r}^{l, m}\left(\psi_{r}^{l+1}-\psi_{r}^{l}\right)\right. \\
& \left.+d_{k, k}^{l, m}\left(\psi_{k}^{l+1}-\psi_{k}^{l}\right)\right), k=1,2, m=1,2,3,
\end{aligned}
$$

where $c_{m}=1$ for $m=1,2$, and $c_{m}=\frac{1}{2}$ for $m=3$. Also, $\psi_{r}^{l}=$ $\frac{\sigma^{2}\left(\left(x^{l}\right)^{2}+\left(y^{l}\right)^{2}+h^{2}\right)}{\beta_{0}}, \psi_{i}^{l}=\frac{\sigma^{2}\left(\left(x^{l}-x_{i}\right)^{2}+\left(y^{l}-y_{i}\right)^{2}+h^{2}\right)}{\beta_{0}}$, for $i=1,2$, and SINR of vehicle $k$ (for $k=1,2$ ) equals $\gamma_{k, l b}^{l, m}=d_{m} p_{r} p_{k}$, where $d_{m}^{-1}=p_{r} \psi_{r}^{l}+\left(p_{1}+p_{2}\right) \psi_{k}^{l}+\psi_{r}^{l} \psi_{k}^{l}+\left(p_{1}+p_{2}\right) p_{r}$, for $m=1,2$, and $d_{m}^{-1}=p_{r} \psi_{r}^{l}+p_{k} \psi_{k}^{l}+\psi_{r}^{l} \psi_{k}^{l}+p_{r} p_{k}$, for $m=3$. Partial derivative of SINR of vehicle $k$ (for $k=1,2$ ) to variable $i \in\{r, k\}$ is denoted by $d_{k, i}^{l, m}[n]$ and equals $d_{k, r}^{l, m}=-d_{m}^{2} p_{r} p_{k}\left(p_{r}+\psi_{k}^{l}\right)$, for $m=1,2,3, d_{k, k}^{l, m}=-d_{m}^{2} p_{r} p_{k}\left(\left(p_{1}+p_{2}\right)+\psi_{r}^{l}\right)$, for $m=1,2$, and $d_{k, k}^{l, m}=-d_{m}^{2} p_{r} p_{k}\left(p_{k}+\psi_{r}^{l}\right)$, for $m=3$.

Proof. See Appendix B.

This proposition shows that for given trajectory and mode coefficients at the $l^{\text {th }}$ iteration, the sum-rate of vehicles at (P1.1) is lower-bounded by the summation of rates in (24). It then follows that the optimal value of (P1.1) is lower-bounded by the optimal value of the following problem

$$
\begin{aligned}
& \text { (P1.2): } \max _{\mathbf{X}^{l+1}, \mathbf{Y}^{l+1}} \sum_{n=1}^{N} \sum_{s=1}^{S} \sum_{k=1}^{K} \alpha_{s, n}^{l} R_{k, \mathrm{lb}}^{l+1,1}[n] \\
& +\beta_{s, n}^{l} R_{k, \mathrm{lb}}^{l+1,2}[n]+\gamma_{s, n}^{l} R_{k, \mathrm{lb}}^{l+1,3}[n] \\
& \text { s.t. } \quad \sum_{s=1}^{S} \alpha_{s, n}^{l} R_{k, l b}^{l+1,1}[n]+\beta_{s, n}^{l} R_{k, l b}^{l+1,2}[n] \\
& +\gamma_{s, n}^{l} R_{k, \mathrm{bb}}^{l+1,3}[n] \geq R_{k}^{t}, \forall n, \forall k, \\
& \text { (15), (16), (17), (18). }
\end{aligned}
$$

Problem (P1.2) is a convex problem which can be efficiently solved by convex optimization techniques such as the interiorpoint method. Note that we proved in Proposition 3 that the objective function of (P1.2) is non-decreasing over iterations and is globally upper-bounded by the optimal value of (P1.1). Therefore, the proposed sub-optimal algorithm converges. One can see the summary of the proposed iterative method for solving (P1.1) in Algorithm 1.

Algorithm 1 Iterative trajectory optimization and operation mode selection for (P1.1) with fixed power allocation.

1: Initialize the UAV trajectory $\mathbf{X}^{l}$ and $\mathbf{Y}^{l}$, and let $l=0$.

2: repeat

3: $\quad$ Find the binary coefficients $\alpha_{s, n}^{l}, \beta_{s, n}^{l}$ and $\gamma_{s, n}^{l}$, for each $n$ based on the UAV trajectory $\mathbf{X}^{l}$ and $\mathbf{Y}^{l}$ and locations of vehicles and BS (refer to Table \).

4: $\quad$ Solve the convex problem (P1.2) by the interior-point method and find the optimal solution $\mathbf{X}^{l+1}$ and $\mathbf{Y}^{l+1}$.

5: $\quad$ Update $l=l+1$.

6: until convergence or a maximum number of iterations is reached.

\section{B. Power Allocation with Fixed Trajectory}

In this subsection, we solve the second sub-problem of (P1) to find optimal values for the power allocation of vehicles at the BS $\left(\mathbf{P}_{1}\right.$ and $\left.\mathbf{P}_{2}\right)$ and the UAV power $\left(\mathbf{P}_{r}\right)$, assuming that the trajectory of the UAV ( $\mathbf{X}$ and $\mathbf{Y}$ ) is fixed. Note that for a fixed trajectory, the operation mode coefficients $\mathbf{A}, \mathbf{B}$, and $\boldsymbol{\Gamma}$ are known. Hence, the optimization problem can be written as

$$
\begin{aligned}
\text { (P1.3): } \max _{\mathbf{P}_{1}, \mathbf{P}_{2}, \mathbf{P}_{\mathbf{r}}} & \sum_{n=1}^{N} \sum_{s=1}^{S} \sum_{k=1}^{K} \alpha_{s, n} R_{k}^{1}[n] \\
& \quad+\beta_{s, n} R_{k}^{2}[n]+\gamma_{s, n} R_{k}^{3}[n] \\
\text { s.t. } & \text { (14), (19), 20), 21), 22). }
\end{aligned}
$$


It is clear that the objective function of (P1.3) in (27) and constraint (14) are not concave functions with respect to $\mathbf{P}_{1}$, $\mathbf{P}_{2}$, and $\mathbf{P}_{r}$. In the following lemmas, we provide two convex functions which can be used for the concave approximation of vehicle rates.

Lemma 2. Consider $x, y$, and $z$ as three variables, and $a$, $b, c, d, e$, and $f$ as constants. Then the function $f(x, y, z)=$ $\log _{2}(a x y+b x z+c x+d y+e z+f)$, is a concave function with respect to $x, y$, and $z$, if $\frac{a}{d}=\frac{b}{e}=\frac{c}{f}$.

Proof. Assuming $\frac{a}{d}=\frac{b}{e}=\frac{c}{f}$, we can show function $\mathrm{f}$ as

$$
\begin{aligned}
f(x, y, z) & =\log _{2}\left(a x y+b x z+c x+d y+\frac{d b}{a} z+\frac{d c}{a}\right) \\
& =\log _{2}\left(x+\frac{d}{a}\right)+\log _{2}(a y+b z+c) .
\end{aligned}
$$

Hence, $f(x, y, z)$ is the summation of two concave functions, and the proof is completed.

Lemma 3. Consider $x$ and $y$ as two variables, and $a, b, c$, and $d$ as constants. Then the function $f(x, y)=\log _{2}(a x y+$ $b x+c y+d)$ is a concave function with respect to $x$ and $y$, if $\frac{a}{c}=\frac{b}{d}$.

Proof. The proof of this lemma is the same with Lemma 2 and hence we remove it.

Lemma 4. The sum-rate of the two vehicles can be approximated in the form of a DC function as

$$
\begin{aligned}
& R_{d c, 1}^{1}+R_{d c, 2}^{1} \approx \log _{2}\left(p_{r} h_{1} h_{r} p_{1}+p_{r} h_{1}+p_{1} h_{r}+1\right) \\
& -\log _{2}\left(p_{r} h_{1}+\left(p_{1}+p_{2}\right) h_{r}+1\right) \\
& +\log _{2}\left(p_{r} h_{2} h_{r}\left(p_{1}+p_{2}\right)+p_{r} h_{2}+\left(p_{1}+p_{2}\right) h_{r}+1\right) \\
& -\log _{2}\left(p_{r} h_{2} h_{r} p_{1}+p_{r} h_{2}+p_{1} h_{r}+1\right), \\
& \quad R_{d c, 1}^{2}+R_{d c, 2}^{2} \approx \\
& \log _{2}\left(p_{r} h_{1} h_{r}\left(p_{1}+p_{2}\right)+p_{r} h_{1}+\left(p_{1}+p_{2}\right) h_{r}+1\right) \\
& \quad-\log _{2}\left(p_{r} h_{1} h_{r} p_{2}+p_{r} h_{1}+p_{2} h_{r}+1\right) \\
& \quad+\log _{2}\left(p_{r} h_{2} h_{r} p_{2}+p_{r} h_{2}+p_{2} h_{r}+1\right) \\
& \quad-\log _{2}\left(p_{r} h_{2}+\left(p_{1}+p_{2}\right) h_{r}+1\right), \\
& \sum_{k=1}^{K} R_{d c, k}^{3}=\sum_{k=1}^{K} \frac{1}{2}\left(\log _{2}\left(p_{r} h_{k} h_{r} p_{k}+p_{r} h_{k}+2 p_{k} h_{r}+2\right)\right. \\
& \left.\quad-\log _{2}\left(p_{r} h_{k}+2 p_{k} h_{r}+2\right)\right),
\end{aligned}
$$

where $R_{d c, k}^{m}$ indicates the DC approximation of vehicle $k$ in mode $m$.

Proof. We can write the sum-rate of the two vehicles at mode $m=1$ from (3) and (4) as

$$
\begin{aligned}
R_{1}^{1} & +R_{2}^{1}=\log _{2}\left(\frac{e_{1}}{f}\right) \\
& +\log _{2}\left(\frac{p_{r} h_{2} h_{r}\left(p_{1}+p_{2}\right)+p_{r} h_{2}+\left(p_{1}+p_{2}\right) h_{r}+1}{e_{2}}\right) \\
& =\log _{2}\left(\frac{e_{1}}{e_{2}}\right) \\
& +\log _{2}\left(\frac{p_{r} h_{2} h_{r}\left(p_{1}+p_{2}\right)+p_{r} h_{2}+\left(p_{1}+p_{2}\right) h_{r}+1}{f}\right),
\end{aligned}
$$

where $e_{k}=p_{r} h_{k} h_{r} p_{1}+p_{r} h_{k}+\left(p_{1}+p_{2}\right) h_{r}+1$, and $f=$ $p_{r} h_{1}+\left(p_{1}+p_{2}\right) h_{r}+1$. According to Lemma 2] the second logarithmic function of second equality in (32) is in the form of the difference of two concave functions as (29). For the first logarithmic function at second equality of (32), we subtract $p_{2} h_{r}$ from the numerator and denominator to approximate it with a DC function as 29). With the same procedure, the sum-rate at $m=2$ can be approximated in the form of a DC function as (30). For $m=3$, from (8) we have $\sum_{k=1}^{K} R_{k}^{3}=\sum_{k=1}^{K} \frac{1}{2} \log _{2}\left(\frac{p_{r} h_{k} h_{r} p_{k}+p_{r} h_{k}+2 p_{k} h_{r}+2}{p_{r} h_{k}+2 p_{k} h_{r}+2}\right)$, which according to Lemma 3 is a DC function as (31). Hence, the proof is completed.

In order to solve (P1.3), we apply the SCA method using the approximated DC sum-rate in Lemma 4. To this end, we find a concave approximation of the DC objective function in the following proposition. Note that the power allocations of the BS and UAV nodes are indicated by $\left(p_{r}^{l}, p_{1}^{l}, p_{2}^{l}\right)$ and $\left(p_{r}^{l+1}, p_{1}^{l+1}, p_{2}^{l+1}\right)$ at the $l^{\text {th }}$ and $(l+1)^{\text {th }}$ iterations, respectively. Also, the lower-bound concave rate of vehicle $k$ at mode $m$ is indicated by $R_{k, \mathrm{lb}}^{l+1, m}$ at each time slot $n$.

Proposition 4. At each time slot n, for any given UAV trajectory and for any power allocation at the $l^{\text {th }}$ iteration $\left(p_{r}^{l}, p_{1}^{l}, p_{2}^{l}\right)$, one approximated concave non-decreasing lowerbound of the rate of vehicle $k$ at mode $m$ and at the $(l+1)^{\text {th }}$ iteration of the SCA method equals

$$
\begin{aligned}
R_{k, l b}^{l+1, m} & =\log _{2}\left(p_{r}^{l+1} h_{k} h_{r} p_{k}^{l+1}+p_{r}^{l+1} h_{k}+p_{k}^{l+1} h_{r}+1\right) \\
& -\log _{2}\left(p_{r}^{l} h_{k}+\left(p_{1}^{l}+p_{2}^{l}\right) h_{r}+1\right) \\
& -d_{k}^{l, m}\left(p_{1}^{l+1}-p_{1}^{l}\right)-t_{k}^{l, m}\left(p_{2}^{l+1}-p_{2}^{l}\right)-c_{k}^{l, m}\left(p_{r}^{l+1}-p_{r}^{l}\right), \\
& (m, k)=(1,1),(m, k)=(2,2),
\end{aligned}
$$

$R_{k, l b}^{l+1, m}=\log _{2}\left(p_{r}^{l+1} h_{k} h_{r}\left(p_{1}^{l+1}+p_{2}^{l+1}\right)+p_{r}^{l+1} h_{k}\right.$

$$
\left.+\left(p_{1}^{l+1}+p_{2}^{l+1}\right) h_{r}+1\right)-\log _{2}\left(p_{r}^{l} h_{k} h_{r} p_{k^{\prime}}^{l}+p_{r}^{l} h_{k}+p_{k^{\prime}}^{l} h_{r}+1\right)
$$$$
-d_{k}^{l, m}\left(p_{1}^{l+1}-p_{1}^{l}\right)-t_{k}^{l, m}\left(p_{2}^{l+1}-p_{2}^{l}\right)-c_{k}^{l, m}\left(p_{r}^{l+1}-p_{r}^{l}\right) \text {, }
$$$$
(m, k)=(1,2),(m, k)=(2,1),
$$

$$
\begin{aligned}
& R_{k, l b}^{l+1, m}=\frac{1}{2}\left(\log _{2}\left(p_{r}^{l+1} h_{k} h_{r} p_{k}^{l+1}+p_{r}^{l+1} h_{k}+2 p_{k}^{l+1} h_{r}+2\right)\right. \\
& -\log _{2}\left(p_{r}^{l} h_{k}+2 p_{k}^{l} h_{r}+2\right) \\
& \left.-d_{k}^{l, m}\left(p_{1}^{l+1}-p_{1}^{l}\right)-t_{k}^{l, m}\left(p_{2}^{l+1}-p_{2}^{l}\right)-c_{k}^{l, m}\left(p_{r}^{l+1}-p_{r}^{l}\right)\right), \\
& (m, k)=(3,1),(m, k)=(3,2),
\end{aligned}
$$

where by assuming $F_{k}=\ln 2\left(p_{r}^{l} h_{k}+\left(p_{1}^{l}+p_{2}^{l}\right) h_{r}+1\right), G_{k}=$ $\ln 2\left(p_{r}^{l} h_{k} h_{r} p_{k^{\prime}}^{l}+p_{r}^{l} h_{k}+p_{k^{\prime}}^{l} h_{r}+1\right)$, and $J_{k}=\ln 2\left(p_{r}^{l} h_{k}+2 p_{k}^{l} h_{r}+\right.$ 2), we have $d_{1}^{l, 1}=\frac{h_{r}}{F_{1}}, t_{1}^{l, 1}=\frac{h_{r}}{F_{1}}, c_{1}^{l, 1}=\frac{h_{1}}{F_{1}}, d_{2}^{l, 2}=\frac{h_{r}}{F_{2}}, t_{2}^{l, 2}=\frac{h_{r}}{F_{2}}$, $c_{2}^{l, 2}=\frac{h_{2}}{F_{2}}, d_{2}^{l, 1}=\frac{p_{r}^{l} h_{2} h_{r}+h_{r}}{G_{2}}, t_{2}^{l, 1}=0, c_{2}^{l, 1}=\frac{p_{1}^{l} h_{2} h_{r}+h_{2}}{G_{2}}, d_{1}^{l, 2}=0$, $t_{1}^{l, 2}=\frac{p_{r}^{l} h_{1} h_{r}+h_{r}}{G_{1}}, c_{1}^{l, 2}=\frac{p_{2}^{l} h_{1} h_{r}+h_{1}}{G_{1}}, d_{1}^{l, 3}=\frac{2 h_{r}}{J_{1}}, t_{1}^{l, 3}=0, c_{1}^{l, 3}=$ $\frac{h_{1}}{J_{1}}, d_{1}^{2,3}=0, t_{2}^{l, 3}=\frac{2 h_{r}}{J_{2}}$, and $c_{1}^{2,3}=\frac{h_{2}}{J_{2}}$.

Proof. According to Lemma 4, the rates of vehicles at (P1.3) can be shown as a DC function. On the other hand, we know that the first-order Taylor series expansion of a convex function 
provides a global lower-bound. Hence, at each iteration $l+1$ of the SCA method, we derive the first-order Taylor expansion of the convex parts of rates around the solution of the previous iteration $l$. The summation of these new affine Taylor approximations of convex parts and concave parts of rates will be a concave function as in (33), (34), and (35). Also, due to maximizing concave approximations at each iteration, the objective value of (P1.3) at the SCA method is non-decreasing with $l$. Hence, the proof is completed.

Proposition 4 shows that for a given power allocation at the $l^{\text {th }}$ iteration, the sum-rate of vehicles at $(\mathrm{P} 1.3)$ is lowerbounded by the summation of rates in (33), (34), and (35). It then follows that the optimal value of (P1.3) is lower-bounded by the optimal value of the following problem

$$
\begin{aligned}
& \text { (P1.4): } \max _{\mathbf{P}_{\mathbf{1}}^{\mathbf{l + 1}}, \mathbf{P}_{\mathbf{2}}^{\mathbf{+}+\mathbf{1}}, \mathbf{P}_{\mathbf{r}}^{\mathbf{l + 1}}} \sum_{n=1}^{N} \sum_{S=1}^{S} \sum_{k=1}^{K} \alpha_{s, n} R_{k, \mathrm{lb}}^{l+1,1}[n] \\
& +\beta_{s, n} R_{k, \mathrm{lb}}^{l+1,2}[n]+\gamma_{s, n} R_{k, \mathrm{lb}}^{l+1,3}[n] \\
& \text { s.t. } \\
& \sum_{s=1}^{S} \alpha_{s, n} R_{k, \mathrm{lb}}^{l+1,1}[n]+\beta_{s, n} R_{k, \mathrm{lb}}^{l+1,2}[n] \\
& +\gamma_{s, n} R_{k, \mathrm{lb}}^{l+1,3}[n] \geq R_{k}^{t h}, \forall n, \forall k, \\
& \text { (19), (20), (21), (22). }
\end{aligned}
$$

Problem (P1.4) is a convex problem. Note that we proved in Proposition 4 that the objective function of (P1.4) is nondecreasing over iterations and is globally upper-bounded by the optimal value of (P1.3). Therefore, the proposed suboptimal algorithm converges. One can see the summary of the proposed iterative method for solving (P1.3) in Algorithm 2 .

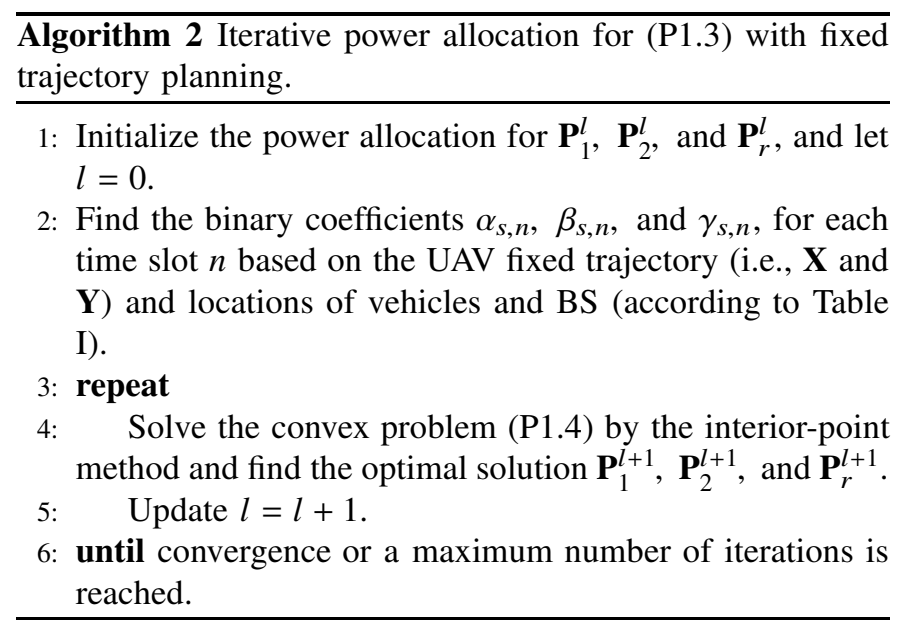

\section{Iterative Power Allocation and Trajectory Planning}

In this subsection, we apply the AO method to solve the problem (P1). To this end, we solve the trajectory planning, operation mode selection, and power allocation sub-problems alternatively, i.e., at each iteration we first optimize the trajectory and determine operation mode coefficients. Then, using this trajectory and operation mode coefficients, we optimize $\overline{\text { Algorithm } 3 \text { Iterative power allocation, trajectory planning }}$ and mode selection for P1.

1: Initialize power allocation $\mathbf{P}_{1}^{l}, \quad \mathbf{P}_{2}^{l}$, and $\mathbf{P}_{r}^{l}$ and UAV trajectory $\mathbf{X}^{l}$ and $\mathbf{Y}^{l}$, and let $l=0$.

2: Find the binary coefficients $\alpha_{s, n}^{0}, \beta_{s, n}^{0}$ and $\gamma_{s, n}^{0}$, for each time slot $n$ based on the UAV initial trajectory (i.e., $\mathbf{X}^{0}$ and $\mathbf{Y}^{0}$ ) and locations of vehicles and BS (according to Table (1).

3: repeat

4: $\quad$ Solve the convex problem (P1.2) with fixed power allocations $\mathbf{P}_{1}^{l}, \mathbf{P}_{2}^{l}$, and $\mathbf{P}_{r}^{l}$ by the interior-point method, and find the optimal solution for $\mathbf{X}^{l+1}$ and $\mathbf{Y}^{l+1}$.

5: $\quad$ Update the binary coefficients $\alpha_{s, n}^{l+1}, \beta_{s, n}^{l+1}$ and $\gamma_{s, n}^{l+1}$, for each time slot $n$ based on the new UAV trajectory (i.e., $\mathbf{X}^{l+1}$ and $\mathbf{Y}^{l+1}$ ) according to Table $\Pi$

6: Solve the convex problem (P1.4) with the fixed UAV trajectory $\mathbf{X}^{l+1}$ and $\mathbf{Y}^{l+1}$ by the interior-point method, and find the optimal solution for $\mathbf{P}_{1}^{l+1}, \mathbf{P}_{2}^{l+1}$, and $\mathbf{P}_{r}^{l+1}$.

7: $\quad$ Update $l=l+1$.

8: until convergence or a maximum number of iterations is reached.

the power allocation sub-problem. The associated algorithm is summarized in Algorithm 3.

According to Proposition 3 and Proposition 4 the optimal value of original problem $(\mathrm{P} 1)$ is a global upper-bound for the optimal values of sub-problems (P1.2) and (P1.4), and hence it is also an upper-bound for the optimal value of Algorithm 3. Also, since Algorithm 3 performs Algorithm 1 and Algorithm 2 alternatively, the objective value of Algorithm 3 is non-decreasing with $l$. As a result, the proposed suboptimal method at Algorithm 3 is guaranteed to converge.

\section{Computational Complexity Analysis}

Now, the computational complexity of proposed iterative solutions for P1 in Algorithms 1, 2 and 3 is presented. At each iteration $l$ of AO method in Algorithm 1 and 2, computational complexity is dominated by solving convex problems in (P1.2) and (P1.4), respectively. These convex problems are solved using the interior point method. According to [36], the interior point method method requires $\log \left(\frac{n_{c}}{t^{0} \varrho}\right) / \log \varepsilon$ number of iterations (Newton steps) to solve a convex problem, where $n_{c}$ is the total number of constraints, $t^{0}$ is the initial point for approximating the accuracy of the interior-point method, $0<\varrho \ll 1$ is the stopping criterion, and $\varepsilon$ is used for updating the accuracy of the interior point method. For (P1.2) and (P1.4), number of constraints are $n_{c}^{t}=7 N+1$ and $n_{c}^{p}=6 N+2$, respectively. Note that superscripts $t$ (for trajectory) and $p$ (for power) indicate problems (P1.2) and (P1.4), respectively. Hence, the computational complexity of Algorithm 1 and 2 will be $O\left(N_{L}^{t}\left(\frac{\log \left(\frac{N}{t^{0, t} \varrho^{t}}\right)}{\log \varepsilon^{t}}\right)\right)$ and $O\left(N_{L}^{p}\left(\frac{\log \left(\frac{N}{t^{0, p} \varrho^{p}}\right)}{\log \varepsilon^{p}}\right)\right)$, where $N_{L}^{t}$ and $N_{L}^{p}$ are the number of iterations for convergence of Algorithm 1 and 2, respectively. At each iteration $l$ of Algorithm 3, computational complexity is dominated by solving two convex problems in $(\mathrm{P} 1.2)$ and $(\mathrm{P} 1.4)$ and hence, it will be 
$O\left(N_{L}\left(\frac{\log \left(\frac{N}{t^{0, t} \varrho^{t}}\right)}{\log \varepsilon^{t}}+\frac{\log \left(\frac{N}{t^{0, p} \rho^{p}}\right)}{\log \varepsilon^{p}}\right)\right)$, where $N_{L}$ is the number of iterations for convergence of Algorithm 3.

\section{Minimum-Rate Maximization}

In this section, we aim to maximize a new objective function in which the throughput fairness between vehicles and among different time slots is considered. Indeed, in delay-sensitive applications, we have to consider fairness and hence maximize the min-rate of vehicles. Note that based on Proposition 2. OMA has better min-rate performance in comparison to NOMA at high SNR regime, and hence we select OMA mode $(m=3)$ for multiple access scheme. Assuming the same parameters with $(\mathrm{P} 1)$, the optimization problem can be formulated as
(P2):

$$
\max _{\mathbf{P}_{1}, \mathbf{P}_{2}, \mathbf{P}_{r}, \mathbf{X}, \mathbf{Y}} \min _{n \in \mathcal{N}, k \in \mathcal{K}} R_{k}^{3}[n]
$$

s.t. where $\mathcal{N}=\{1,2, \ldots, N\}$, and $\mathcal{K}=\{1,2\}$. The objective function of $(\mathrm{P} 2)$ is not concave with respect to trajectory and power variables. Hence, (P2) is a non-convex problem. In order to solve this problem, as with the solution to problem (P1), we use the AO method. For the trajectory planning subproblem with fixed power allocations, the objective function of (P2) is still a non-concave function. Therefore, we apply the SCA method in which we utilize the concave lowerbound approximation of vehicle rates derived in Proposition 3 for each iteration $l+1$ of the SCA method. Given that the minimum of concave functions is a concave function, the approximated objective function of (P2) is concave. For the power allocation sub-problem with fixed trajectory, the objective function of (P2) is not concave, and we apply the SCA method in which utilizing Proposition 4 we derive concave approximations for the rate of each vehicle. Due to the similarity of derivations with Section IV, we do not mention these derivations in this section. Finally, we apply the AO method as in Algorithm 3, to solve the problem (P2).

\section{NUMERICAL RESULTS}

In this section, numerical results are provided to validate the proposed algorithms for trajectory planning and power allocation. The following default parameters are applied in the simulations except that we specify different values for them. We assume that the BS is located at the origin and the UAV starts and ends its flight at the same location $\left(x_{s}, y_{s}\right)=$ $\left(x_{f}, y_{f}\right)=(200,300)$. Note that in this paper, the units of $x$ and $y$ in $(x, y)$ are in meters. We assume that UAV flight ranges are $\left(x_{\min }, x_{\max }\right)=(0,1000)$ and $\left(y_{\min }, y_{\max }\right)=(0,1000)$. The default velocity for the UAV is $v=30 \mathrm{~m} / \mathrm{s}$, and the UAV flies at fixed height $h=100 \mathrm{~m}$. There are two vehicles in our system that move along a two-lane street in parallel with $\mathrm{y}$ axis. Vehicle 1 and vehicle 2 start their path at $\left(x_{1}[0], y_{1}[0]\right)=$ $(700,100)$ and $\left(x_{2}[0], y_{2}[0]\right)=(702,0)$, respectively. Vehicles move with the fixed velocity $v_{1}=v_{2}=15 \mathrm{~m} / \mathrm{s}$. The communication bandwidth is $B=10 \mathrm{MHz}$ with a carrier frequency at $5 \mathrm{GHz}$, and a noise power spectrum density of $N_{0}=-174 \mathrm{dBm} / \mathrm{Hz}$. The reference SNR at the distance

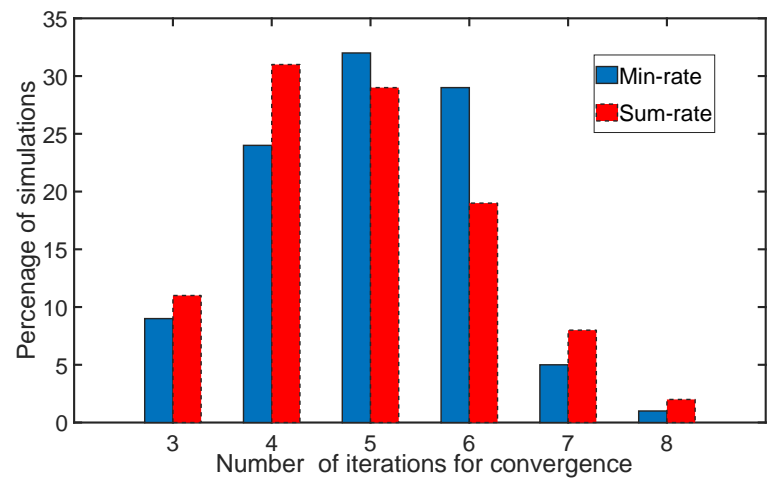

(a) Histogram of the number of iterations for convergence

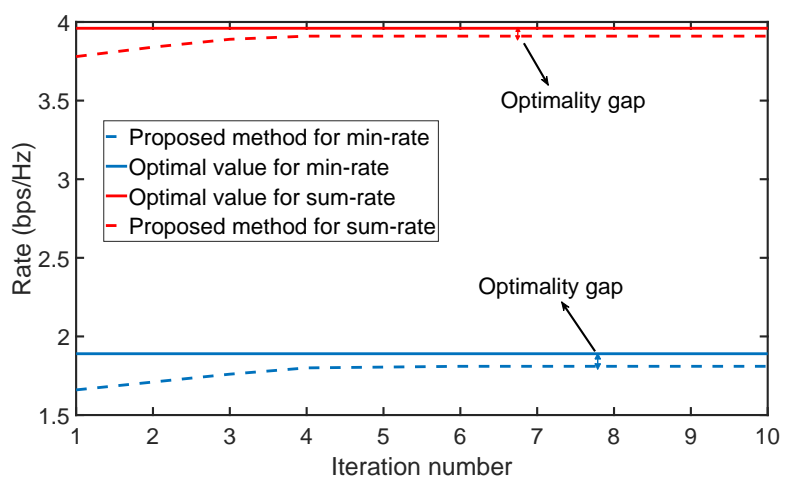

(b) Optimality gap

Fig. 5: Histogram of the number of iterations for convergence, and performance gap between proposed sub-optimal methods and optimal values for sum-rate and min-rate problems.

$d_{0}=1 \mathrm{~m}$ equals $\frac{\beta_{0}}{B N_{0}}=70 \mathrm{~dB}$. We set the number of time slots as $N=600$ and assume that each time slot equals $\tau=0.1 \mathrm{~s}$. The threshold superiority rate at Table I has default value $R_{\mathrm{th}}^{S}=0.1 \mathrm{bps} / \mathrm{Hz}$. Also, we assume two vehicles have the same minimum rate requirements $R_{1}^{t}=R_{2}^{t}=1 \mathrm{bps} / \mathrm{Hz}$. According to this rate requirement, the default value for the maximum transmit power of the BS and UAV equals $\bar{P}_{s}=\bar{P}_{r}=0.5 \mathrm{~W}$.

In order to solve $\mathrm{P} 1$ and $\mathrm{P} 2$, we should find a feasible initial values for power allocations and the trajectory of the UAV. For this end, we assume that the BS power is allocated equally between two vehicles and among different time slots, i.e., $p_{1}[n]=p_{2}[n]=0.5 \bar{P}_{s}, \forall n$. Also, we assume that the UAV power is equally allocated among different time slots, i.e., $p_{r}[n]=\bar{P}_{r}, \forall n$. In order to find an initial feasible trajectory, we consider straight line between the start and end locations of the UAV as an initial trajectory. With the proposed initial power allocations and trajectory, all of the constraints of $\mathrm{P} 2$ are satisfied and hence, these initial points are in feasible region of $\mathrm{P} 2$. For P1, we have one more constraint rather than $\mathrm{P} 2$, i.e., constraint (14), which guarantees the minimum target rate $R_{k}^{t}$ for vehicle $k$ at time slot $n$. We use the allocated powers and trajectory derived from solving $\mathrm{P} 2$ as an initial point of P1.

Fig. 5 shows the histogram of the number of iterations for the convergence of the proposed algorithms and performance gap between these sub-optimal methods and optimal values. In 


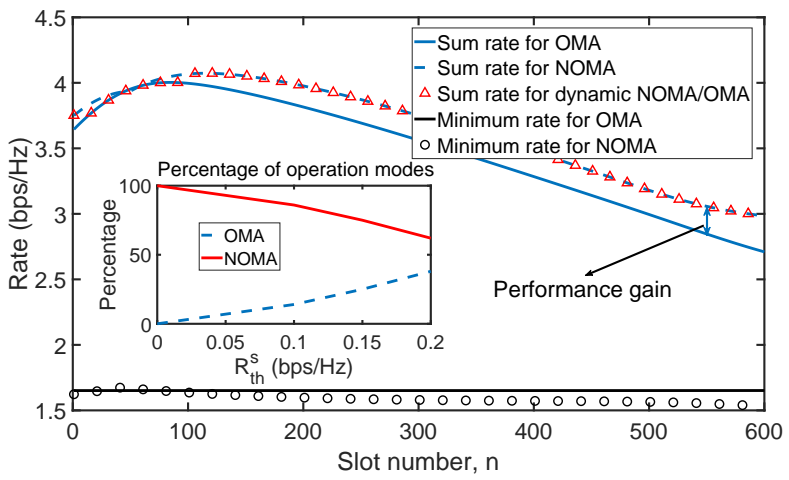

(a) Sum-rate and min-rate with dynamic NOMA/OMA

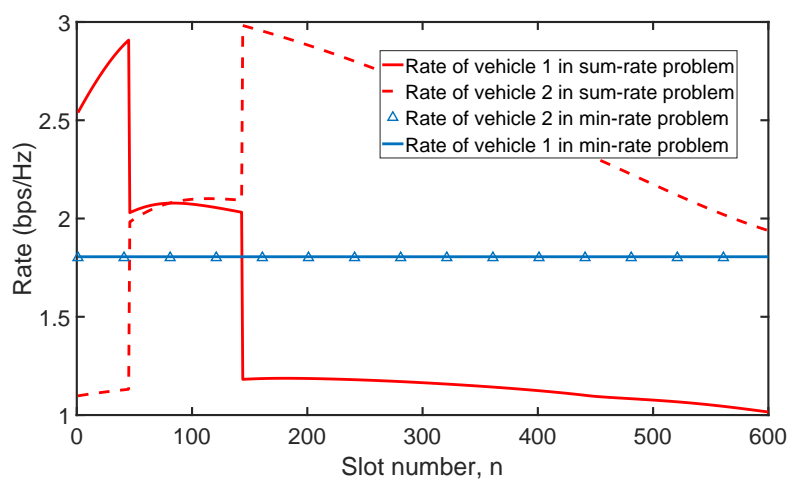

(b) Rates of vehicles for min-rate and sum-rate problems

Fig. 6: Achievable rates with the dynamic NOMA/OMA for the sum-rate and min-rate problems versus slot number $n$.

Fig. 5a, we see that the objective functions of (P.1) and (P.2) in (13) and (38) converge in a few iterations which shows the efficiency of proposed algorithms. For optimization problem (P1), the convergence histogram of Algorithm 3 has been plotted at this figure. Note that Algorithms 1 and 2 which solve problems (P1.1) and (P1.3) are special cases of Algorithm 3 when power allocation and trajectory are fixed, respectively. Also, note that this histogram is based on convergence results of 100 simulations for $N=600$ time slots. In Fig. 5b we can see the performance gap between the proposed suboptimal algorithms and the optimal values for sum-rate and min-rate problems. Also, this figure indicates the convergence of the proposed algorithms in a few iterations. In order to attain optimal values in (P1) and (P2), we perform exhaustive search over all of the feasible values for power allocation coefficients and UAV trajectory. Because a two-dimensional search for UAV trajectory over $N$ time slots is very complex, we consider a small scale case in which we search for the optimal location for UAV placement in the XY-plane. For this end, first, we discretize the power ranges in (19) and (20) and the UAV flight range in (18). Then, we find the optimal power allocation and UAV deployment location that maximizes sum-rate and min-rate problems. In this figure, we assume that $\left(y_{1}, y_{2}\right)=(400,500)$. We can see in this figure that the proposed sub-optimal algorithms for (P1) and (P2) have small performance gaps with optimal values.

Fig. 6 shows the achievable rates with the OMA, NOMA, and dynamic NOMA/OMA schemes for the sum-rate and minrate problems versus slot number $n$. Fig. 6a shows the sumrate and min-rate of vehicles at $N=600$ time slots. In this figure, we assume that vehicle 1 and vehicle 2 start their path at $\left(x_{1}[0], y_{1}[0]\right)=(700,300)$ and $\left(x_{2}[0], y_{2}[0]\right)=(702,0)$, respectively. We can see that for the sum-rate problem, because two vehicles have similar distances to the UAV at initial time slots, OMA has close sum-rate performance with NOMA. Hence, our dynamic scheme selects OMA because of its lower decoding complexity. The channels of vehicles to UAV are degraded at middle and final time slots which leads to superior sum-rate performance of NOMA in comparison to OMA. Our dynamic scheme selects NOMA mode for these time slots. In this figure, we can also see the percentage of operation modes (i.e., OMA or NOMA modes) versus superiority rate $\left(R_{t h}^{S}\right)$. We see that by increasing $R_{t h}^{S}$, the proposed dynamic scheme selects OMA scheme at more time slots. This leads to less complexity (due to lack of SIC at OMA scheme) at vehicles with compromising more sum-rate. We can also see that min-rate of OMA is superior to NOMA. Fig. 6b shows the achievable rates of each vehicle at $N=600$ time slots. For the sum-rate problem, the rates of vehicles in the initial time slots are much better than in the final time slots, and this is due to the existence of better communication links. In the min-rate problem, the rates of the two vehicles are identical at all of the time slots in order to have fairness. Note that OMA mode is applied for the min-rate problem.

Fig. 7 indicates the average sum-rate and min-rate of vehicles versus transmit power for the sum-rate (P1) and minrate (P2) problems, respectively. We assumed that both the BS and UAV have the same transmit power. Fig. $7 \mathrm{a}$ shows the sum-rate of vehicles averaged over $N=600$ time slots. In Fig. 7a, one can see that when vehicles are close to each other at their default paths, less gain is achieved by applying NOMA rather than OMA. This observation matches with the results of Proposition 11 In Fig. 7a, we also assume a scenario that vehicle 1 and vehicle 2 are in two different streets and they start their path at $\left(x_{1}[0], y_{1}[0]\right)=(600,0)$ and $\left(x_{2}[0], y_{2}[0]\right)=(900,0)$, respectively. One can see that we obtain more gain from NOMA in comparison to OMA in this case. We can also see that the proposed dynamic NOMA/OMA scheme has the same sum-rate performance with NOMA. Note that proposed dynamic NOMA/OMA has less decoding complexity than NOMA. We also see the case where the BS-to-UAV link is worse than the UAV-to-vehicle links. We assume that the UAV is located at a fixed position $(650,500)$ in order to have weak channel with the BS. We also assume that the vehicles move along two different streets. We can see that, matching with Proposition 1 in spite of the big difference between channels of vehicles and because of weak links between the BS and UAV, the NOMA and OMA schemes has a close sum-rate performance. Fig. $7 \mathrm{~b}$ shows the min-rate of vehicles during all $N$ time slots. We can see that OMA has better performance than NOMA at the high SNR regime which matches with the results of Proposition 2 . We can also see the min-rate of vehicles with the NOMA scheme for the case where only the power allocation or trajectory of the UAV is optimized. We see that the min-rate of these cases 


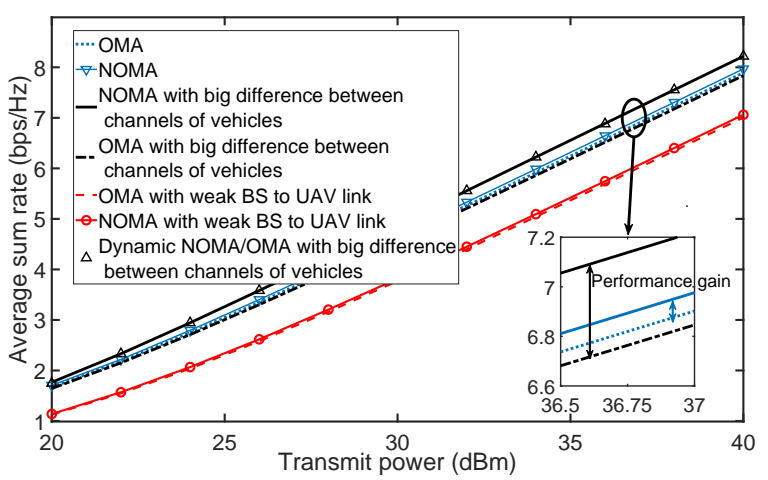

(a) Average sum-rate for different channel gains of two vehicles, and weak BS-to-UAV link.

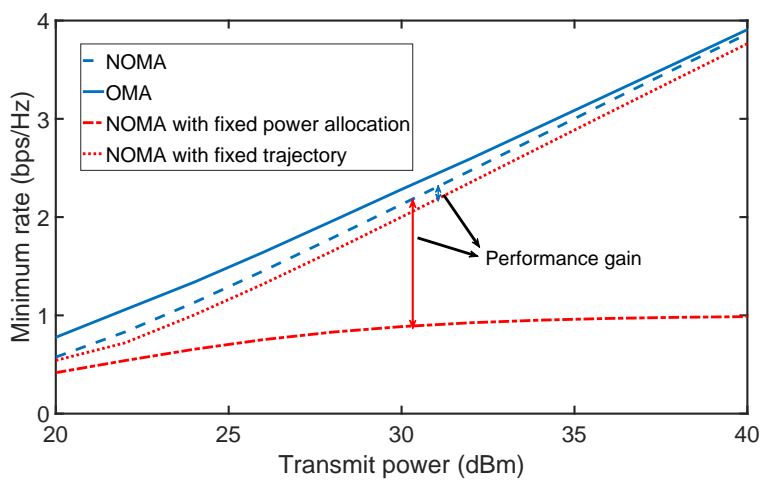

(b) Minimum rate for the cases where power allocation and/or trajectory are optimized.

Fig. 7: Sum-rate and min-rate of vehicles versus transmit power with NOMA/OMA.

is worse than the case where both trajectory and power are optimized. In the NOMA scheme, interference of the strong vehicle over the weak vehicle leads to saturation of the weak vehicle rate at the high SNRs. Hence, we see that the min-rate of the fixed power allocation case tends to a fixed value by increasing transmit power.

Fig. 8 shows the optimized trajectory of the UAV and the path of vehicles at $N=600$ time slots for different scenarios. In Fig. 8a, one can see two dimensional evolution of the $\mathrm{UAV}$ trajectory for the min-rate problem at different iterations of proposed algorithm. At the converged trajectory, the UAV goes forward to be close to the vehicles at middle time slots. This causes fairness in rates of vehicles between initial and middle time slots. Then, the UAV goes up to follow vehicles and provide fair rates for final time slots. Fig. $8 \mathrm{~b}$ indicates the trajectory of UAV for different scenarios of the sum-rate problem. For the case that initial and final locations of UAV flight are the same (i.e., $\left.\left(x_{s}, y_{s}\right)=\left(x_{f}, y_{f}\right)=(200,300)\right)$, the UAV flies many time slots over the line $x=356 \mathrm{~m}$ in which vehicles have better sum-rate. We can see the trajectory for the case where the initial and final locations of the UAV are different points $\left(x_{s}, y_{s}\right)=(200,300)$ and $\left(x_{f}, y_{f}\right)=(550,550)$, respectively. With these locations and velocity $v=30 \mathrm{~m} / \mathrm{s}$, the $\mathrm{UAV}$ is interested in spending more time slots over the line $x=356 \mathrm{~m}$. We also solved the deployment problem for the

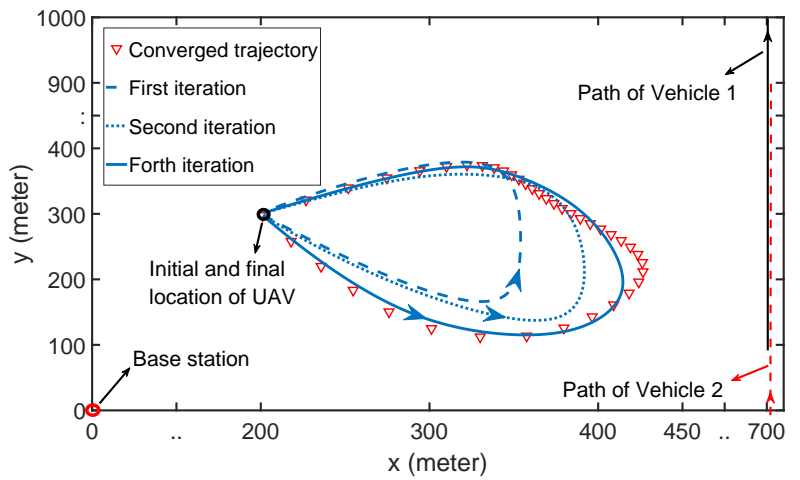

(a) Evolution of UAV trajectory at min-rate problem

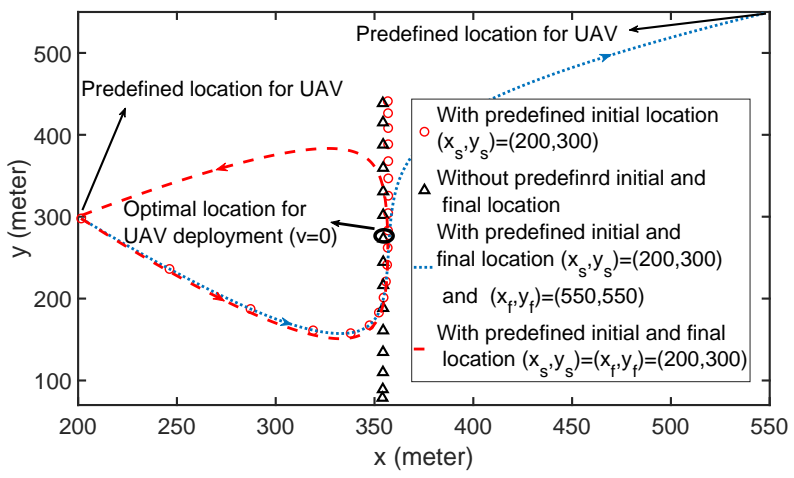

(b) Different scenarios at sum-rate problem

Fig. 8: Trajectory of the UAV for different scenarios. (a) Evolution of UAV trajectory at the min-rate problem. (b) Trajectory for sum-rate problem when the initial and/or final points of flight are not predefined, and for two different velocities of the UAV, i.e., $v=0,30 \mathrm{~m} / \mathrm{s}$.

UAV which is equivalent to the special case of the sum-rate problem where the UAV has no constraint for initial or final locations and its velocity is $v=0 \mathrm{~m} / \mathrm{s}$. In this case, the optimal point for deploying the UAV is $\left(x_{\mathrm{opt}}, y_{\mathrm{opt}}\right)=(354,264)$ which is on the line $x=354 \mathrm{~m}$. In Fig. $8 \mathrm{~b}$ we depicted the trajectory of the UAV for the case where the UAV has no predefined initial or final location. At this case, the UAV only flies over the line $x=354 \mathrm{~m}$ following vehicles. This shows that in contrast to a fixed user case in which the UAV hovers over the best deployment location, in vehicular networks, the UAV tends to follow the vehicles to make the best performance. Finally, one can see the trajectory of the UAV when the UAV has to start its flight from a predefined location. In this case, the UAV goes to the line $x=356 \mathrm{~m}$ and flies over this line towards vehicles.

Fig. 9 indicates the allocated power with the dynamic NOMA/OMA scheme for each vehicle at the BS and UAV during the flight time. We can see these allocated powers for the sum-rate and min-rate problems with default parameters at Fig. 9a. For the min-rate problem, it is clear that in order to have fair rates among different time slots, the UAV and BS consume much power at final time slots in which vehicles are far from the UAV. We can also see power allocation for the sum-rate problem in this figure. For the first time slots, due to 


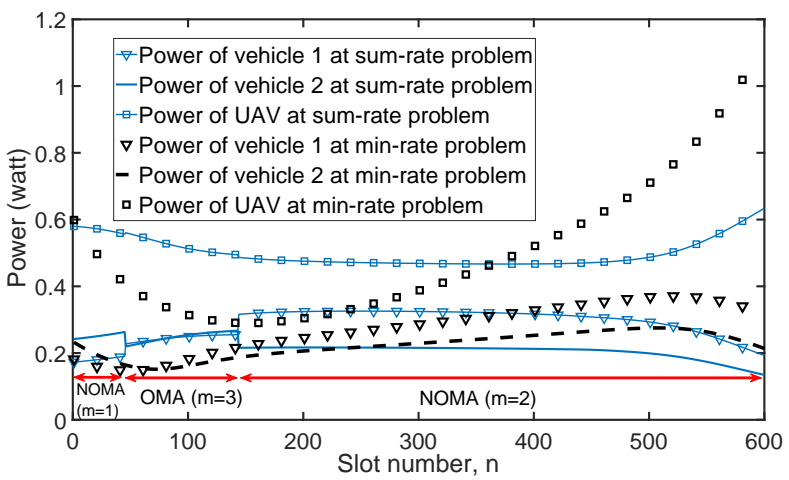

(a) Min/sum-rate problems with default parameters

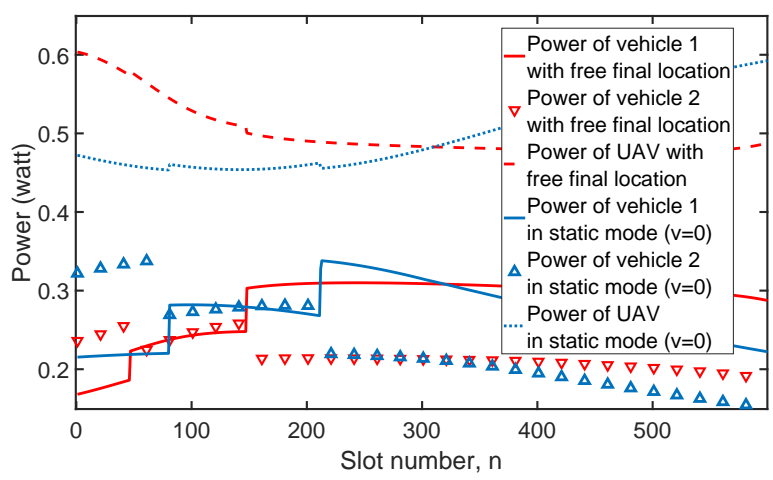

(b) Sum-rate problem with different scenarios.

Fig. 9: Power allocation for the vehicles and the UAV with dynamic NOMA/OMA. (9a) Min-rate and sum-rate problems with default parameters. (9b) Sum-rate problem with static relaying and the case where the UAV has no constraint for its final location.

establishing good links between the UAV and vehicles, more power is allocated to UAV to increases the sum-rate. Fig. $9 \mathrm{~b}$ shows power allocation for the sum-rate problem with different scenarios. For the case where there is no constraint for the final location of the UAV, the UAV consumes more power at initial time slots because at these time slots the vehicles are close to the UAV. We also depicted the allocated power for the static case in which the UAV has deployed at the optimal position $\left(x_{\mathrm{opt}}, y_{\mathrm{opt}}\right)=(354,264)$. We see in Fig. 9b that the BS consumes more power at initial time slots. The reason is that at these time slots UAV-to-vehicle channels have better links and by allocating more power at the BS, better sum-rate can be achieved by the whole system.

\section{CONCLUSION}

In this paper, power allocation and trajectory planning of a drone assisted NR-V2X network with dynamic NOMA/OMA has been investigated. We showed that in a two-user network with a dedicated AF relay, NOMA always has better or equal sum-rate performance in comparison to OMA at high SNR regime. Due to the complexity of SIC decoding at NOMA, we proposed a dynamic NOMA/OMA scheme in which the OMA mode is selected when applying NOMA has only negligible gain. We have formulated two optimization problems which maximize the sum-rate and min-rate of the two vehicles. These optimization problems were not convex, and hence we applied the AO and SCA methods to find power allocation and trajectory of the UAV separately. Simulation results showed that the proposed dynamic scheme can achieve less decoding complexity in comparison to NOMA with a negligible sumrate compromise.

\section{APPENDIX A PROOF OF LEMMA 1}

In order to prove that $f$ is convex, we calculate its first and second order derivatives as

$$
\begin{gathered}
\nabla f=\left[\begin{array}{l}
\frac{\partial f}{\partial x} \\
\frac{\partial f}{\partial y}
\end{array}\right]=\left[\begin{array}{c}
\frac{-(a+c y)}{u^{2}} \\
\frac{-(b+c x)}{u^{2}}
\end{array}\right], \\
\nabla^{2} f=\left[\begin{array}{cc}
\frac{\partial f^{2}}{\partial x^{2}} & \frac{\partial f^{2}}{\partial y \partial x} \\
\frac{\partial f^{2}}{\partial x \partial y} & \frac{\partial f^{2}}{\partial y^{2}}
\end{array}\right]=\frac{1}{u^{3}}\left[\begin{array}{cc}
2(a+c y)^{2} & v \\
v & 2(b+c x)^{2}
\end{array}\right],
\end{gathered}
$$

where $u=a x+b y+c x y+d$ and $v=a c x+c b y+$ $c^{2} x y+2 a b-c d$. In order to show that function $f$ is a convex function, we must prove that $\nabla^{2} f$ is a positive definite matrix (i.e., $\mathbf{t} \nabla^{2} f \mathbf{t}^{T}>0$ for every nonzero vector $\left.\mathbf{t}=\left[\begin{array}{ll}t_{1} & t_{2}\end{array}\right]\right)$. Performing some calculations leads to $\mathbf{t} \nabla^{2} f \mathbf{t}^{T}=$ $\frac{2(a+c y)^{2} t_{1}^{2}+2(b+c x)^{2} t_{2}^{2}+2\left(a c x+c b y+c^{2} x y+2 a b-c d\right) t_{1} t_{2}}{u^{3}}$. For writing the numerator of this equation in the square form we must have $a b=c d$. Then, we have

$\mathbf{t} \nabla^{2} f \mathbf{t}^{T}=\frac{\left(t_{1}(a+c y)+t_{2}(b+c x)\right)^{2}+(a+c y)^{2} t_{1}^{2}+(b+c x)^{2} t_{2}^{2}}{u^{3}}$

One can see that 410 is always positive for $u=a x+b y+$ $c x y+d>0$. For inverse proof, assuming $a b=c d$, we can show function $\mathrm{f}$ as $f(x, y)=\frac{1}{\left(\left(\frac{c}{b}\right) x+1\right)(b y+d)}$. We know that the function $g(x, y)=\frac{1}{x y}$ is a convex function for $x y>0$. On the other hand, the composition of a function with a linear function does not change convexivity. Hence, $f(x, y)=g\left(\left(\frac{c}{b}\right) x+1, b y+\right.$ $d$ ) is a convex function for $\left(\left(\frac{c}{b}\right) x+1\right)(b y+d)>0$. Therefore, the proof is completed.

\section{APPENDIX B \\ PROOF OF PROPOSITION 3}

Assuming $\psi_{r}=\frac{\sigma^{2}}{h_{r}}=\frac{\sigma^{2}\left(x^{2}+y^{2}+h^{2}\right)}{\beta_{0}}, \psi_{i}=\frac{\sigma^{2}}{h_{i}}=$ $\frac{\sigma^{2}\left(\left(x-x_{i}\right)^{2}+\left(y-y_{i}\right)^{2}+h^{2}\right)}{\beta_{0}}$ for $i=1,2$, and replacing them in vehicle SINR in (3), (4), (5), (6), and (8), the SINR of vehicle $k$ equals

$$
\gamma_{k}^{m}=\frac{p_{r} p_{k}}{p_{r} \psi_{r}+\left(p_{1}+p_{2}\right) \psi_{k}+\psi_{r} \psi_{k}},
$$

for $(k, m)=(1,1),(k, m)=(2,2)$,

$$
\gamma_{k}^{m}=\frac{p_{r} p_{k}}{p_{r} \psi_{r}+\left(p_{1}+p_{2}\right) \psi_{k}+\psi_{r} \psi_{k}+p_{k^{\prime}} p_{r}},
$$

for $(k, m)=(2,1),(k, m)=(1,2)$, and

$$
\gamma_{k}^{m}=\frac{p_{r} p_{k}}{p_{r} \psi_{r}+p_{k} \psi_{k}+\psi_{r} \psi_{k}}
$$

for $(k, m)=(1,3),(k, m)=(2,3)$, where $k^{\prime}$ shows the vehicle other than vehicle $k$. Based on Lemma 1, in order to 
convert the SINRs (42), (43), and (44) to a convex function, we have to add fixed terms $\left(p_{1}+p_{2}\right) p_{r}, p_{k} p_{r}$, and $p_{k} p_{r}$ to the denominators of these equations, respectively. Note that these new SINRs are convex with respect to $\psi_{s r}, \psi_{r 1}$, and $\psi_{r 2}$, though they are not convex with respect to $\mathbf{X}$ and Y. We know that the first-order Taylor series expansion of a convex function $f(z)$ provides a global lower-bound, i.e., $f(z) \geq f\left(z_{0}\right)+\nabla f\left(z_{0}\right)^{T}\left(z-z_{0}\right)$. Considering this point that we have a convex approximation of SINRs with respect to $\psi_{s r}$, $\psi_{r 1}$, and $\psi_{r 2}$, we can approximate them at each iteration $l+1$ by their first-order Taylor series expansion around the solution of previous iteration $l$. Therefore, at the SCA method, the SINR of vehicle $k$ at the $(l+1)^{\text {th }}$ iteration can be approximated by $\gamma_{k, 1 \mathrm{~b}}^{l+1, m} \approx \gamma_{k, \mathrm{lb}}^{l, m}+d_{k, r}^{l, m}\left(\psi_{r}^{l+1}-\psi_{r}^{l}\right)+d_{k, k}^{l, m}\left(\psi_{k}^{l+1}-\psi_{k}^{l}\right)$. It is clear that $\gamma_{k, l \mathrm{~b}}^{l+1, m}$ is concave with respect to $\mathbf{X}^{l+1}$ and $\mathbf{Y}^{l+1}$. Also, the composition of a concave function with a logarithm function is a concave function, and hence, $R_{k, \mathrm{lb}}^{l+1, m}=\log _{2}\left(1+\gamma_{k, 1 \mathrm{~b}}^{l+1, m}\right)$ is concave. Finally, note that due to applying first-order expansion for a convex function, we have $R_{k}^{l+1, m}>R_{k, 1 \mathrm{~b}}^{l+1, m}$, and due to the maximization of a concave function at each iteration $l$, we have $R_{k, \mathrm{lb}}^{l+1, m}>R_{k, \mathrm{lb}}^{l, m}$. Considering this point that Taylor expansion of a function around an initial point is exactly equal to the value of the original function at that point (i.e., $R_{k, \mathrm{~b}}^{l, m}=R_{k}^{l, m}$ ), we can conclude $R_{k}^{l+1, m}>R_{k}^{l, m}$. Hence, the rates $R_{k}^{l, m}$ are non-decreasing with $l$ and the proof is completed.

\section{REFERENCES}

[1] K. P. Valavanis and G. J. Vachtsevanos, "Future of unmanned aviation," in Handbook of Unmanned Aerial Vehicles. Springer, 2015, pp. 29933009.

[2] X. Lin, V. Yajnanarayana, S. D. Muruganathan, S. Gao, H. Asplund, H. Maattanen, M. Bergstrom, S. Euler, and Y. E. Wang, "The sky is not the limit: LTE for unmanned aerial vehicles," IEEE Communications Magazine, vol. 56, no. 4, pp. 204-210, Apr. 2018.

[3] R. I. Bor-Yaliniz, A. El-Keyi, and H. Yanikomeroglu, "Efficient 3-D placement of an aerial base station in next generation cellular networks," in 2016 IEEE International Conference on Communications (ICC), May 2016, pp. 1-5.

[4] Y. Zeng, R. Zhang, and T. J. Lim, "Throughput maximization for UAVenabled mobile relaying systems," IEEE Transactions on Communications, vol. 64, no. 12, pp. 4983-4996, Dec. 2016.

[5] P. Zhan, K. Yu, and A. L. Swindlehurst, "Wireless relay communications with unmanned aerial vehicles: Performance and optimization," IEEE Transactions on Aerospace and Electronic Systems, vol. 47, no. 3, pp. 2068-2085, Jul. 2011

[6] G. Araniti, C. Campolo, M. Condoluci, A. Iera, and A. Molinaro, "LTE for vehicular networking: A survey," IEEE Communications Magazine, vol. 51, no. 5, pp. 148-157, May 2013.

[7] H. T. Cheng, H. Shan, and W. Zhuang, "Infotainment and road safety service support in vehicular networking: From a communication perspective," Mechanical Systems and Signal Processing, vol. 25, no. 6, pp. 2020-2038, Aug. 2011.

[8] A. Vinel, "3GPP LTE Versus IEEE 802.11p/WAVE: Which technology is able to support sooperative vehicular safety applications?" IEEE Wireless Communications Letters, vol. 1, no. 2, pp. 125-128, Apr. 2012.

[9] L. Liang, G. Y. Li, and W. Xu, "Resource allocation for D2D-enabled vehicular communications," IEEE Transactions on Communications, vol. 65, no. 7, pp. 3186-3197, Jul. 2017.

[10] G. Liu, Z. Wang, J. Hu, Z. Ding, and P. Fan, "Cooperative NOMA broadcasting/multicasting for low-latency and high-reliability 5G cellular V2X communications," IEEE Internet of Things Journal, vol. 6, no. 5, pp. 7828-7838, Oct. 2019.

[11] G. Naik, B. Choudhury, and J. Park, "IEEE 802.11bd and 5G NR V2X: Evolution of radio access technologies for V2X communications," IEEE Access, vol. 7, pp. $70169-70$ 184, 2019.
[12] Y. Saito, Y. Kishiyama, A. Benjebbour, T. Nakamura, A. Li, and K. Higuchi, "Non-orthogonal multiple access (NOMA) for cellular future radio access," in 2013 IEEE 77th Vehicular Technology Conference (VTC Spring), Jun. 2013, pp. 1-5.

[13] Z. Ding, F. Adachi, and H. V. Poor, "The application of MIMO to non-orthogonal multiple access," IEEE Transactions on Wireless Communications, vol. 15, no. 1, pp. 537-552, Jan. 2016.

[14] Z. Ding, M. Peng, and H. V. Poor, "Cooperative non-orthogonal multiple access in 5G systems," IEEE Communications Letters, vol. 19, no. 8, pp. 1462-1465, Aug. 2015.

[15] Y. Liu, Z. Ding, M. Elkashlan, and H. V. Poor, "Cooperative nonorthogonal multiple access with simultaneous wireless information and power transfer," IEEE Journal on Selected Areas in Communications, vol. 34, no. 4, pp. 938-953, Apr. 2016.

[16] O. Abbasi and A. Ebrahimi, "Cooperative NOMA with full-duplex amplify-and-forward relaying," Transactions on Emerging Telecommunications Technologies, vol. 29, no. 7, p. e3421, Jul. 2018.

[17] Z. Ding, H. Dai, and H. V. Poor, "Relay selection for cooperative NOMA," IEEE Wireless Communications Letters, vol. 5, no. 4, pp. 416419, Aug. 2016

[18] J. Kim and I. Lee, "Non-orthogonal multiple access in coordinated direct and relay transmission," IEEE Communications Letters, vol. 19, no. 11 pp. 2037-2040, Nov. 2015.

[19] O. Abbasi, A. Ebrahimi, and N. Mokari, "NOMA inspired cooperative relaying system using an AF relay," IEEE Wireless Communications Letters, vol. 8, no. 1, pp. 261-264, Feb. 2019.

[20] D. H. Choi, S. H. Kim, and D. K. Sung, "Energy-efficient maneuvering and communication of a single UAV-based relay," IEEE Transactions on Aerospace and Electronic Systems, vol. 50, no. 3, pp. 2320-2327, Jul. 2014.

[21] S. Rohde, M. Putzke, and C. Wietfeld, "Ad hoc self-healing of OFDMA networks using UAV-based relays," Ad Hoc Networks, vol. 11, no. 7, pp. 1893-1906, Sep. 2013.

[22] S. Zhang, H. Zhang, Q. He, K. Bian, and L. Song, "Joint trajectory and power optimization for UAV relay networks," IEEE Communications Letters, vol. 22, no. 1, pp. 161-164, Jan. 2018.

[23] X. Jiang, Z. Wu, Z. Yin, and Z. Yang, "Joint power and trajectory design for UAV-relayed wireless systems," IEEE Wireless Communications Letters, vol. 8, no. 3, pp. 697-700, Jun. 2019.

[24] — "Power and trajectory optimization for UAV-enabled amplify-andforward relay networks," IEEE Access, vol. 6, pp. 48 688-48 696, 2018.

[25] Y. Liu, Z. Qin, Y. Cai, Y. Gao, G. Y. Li, and A. Nallanathan, "UAV communications based on non-orthogonal multiple access," IEEE Wireless Communications, vol. 26, no. 1, pp. 52-57, Feb. 2019.

[26] P. K. Sharma and D. I. Kim, "UAV-enabled downlink wireless system with non-orthogonal multiple access," in 2017 IEEE Globecom Workshops (GC Wkshps), Dec. 2017, pp. 1-6.

[27] M. F. Sohail, C. Y. Leow, and S. Won, "Non-orthogonal multiple access for unmanned aerial vehicle assisted communication," IEEE Access, vol. 6, pp. 22716-22 727, 2018.

[28] A. A. Nasir, H. D. Tuan, T. Q. Duong, and H. V. Poor, "UAV-enabled communication using NOMA," IEEE Transactions on Communications, vol. 67 , no. 7, pp. 5126-5138, Jul. 2019.

[29] X. Liu, J. Wang, N. Zhao, Y. Chen, S. Zhang, Z. Ding, and F. R. $\mathrm{Yu}$, "Placement and power allocation for NOMA-UAV networks," IEEE Wireless Communications Letters, vol. 8, no. 3, pp. 965-968, Jun. 2019.

[30] N. Zhao, X. Pang, Z. Li, Y. Chen, F. Li, Z. Ding, and M. Alouini, "Joint trajectory and precoding optimization for UAV-assisted NOMA networks," IEEE Transactions on Communications, vol. 67, no. 5, pp. 3723-3735, May 2019.

[31] H. Zheng, H. Li, S. Hou, and Z. Song, "Joint resource allocation with weighted max-min fairness for NOMA-enabled V2X communications," IEEE Access, vol. 6, pp. 65 449-65 462, Oct. 2018.

[32] S. Gurugopinath, P. C. Sofotasios, Y. Al-Hammadi, and S. Muhaidat, "Cache-aided non-orthogonal multiple access for 5G-enabled vehicular networks," IEEE Transactions on Vehicular Technology, vol. 68, no. 9, pp. 8359-8371, Sep. 2019.

[33] Z. Ding, P. Fan, and H. V. Poor, "Impact of user pairing on 5G nonorthogonal multiple-access downlink transmissions," IEEE Transactions on Vehicular Technology, vol. 65, no. 8, pp. 6010-6023, Aug. 2016.

[34] C. S. Patel, G. L. Stuber, and T. G. Pratt, "Statistical properties of amplify and forward relay fading channels," IEEE Transactions on Vehicular Technology, vol. 55, no. 1, pp. 1-9, Jan. 2006.

[35] C. Yan, A. Harada, A. Benjebbour, Y. Lan, A. Li, and H. Jiang, "Receiver design for downlink non-orthogonal multiple access (NOMA)," in 2015 
IEEE 81st Vehicular Technology Conference (VTC Spring), May 2015, pp. $1-6$.

[36] S. Boyd and L. Vandenberghe, Convex optimization. Cambridge University Press, 2004 